\title{
The crystal structures of sintered copper nanoparticles: a molecular dynamics study
}

\author{
Bingqing Cheng and Alfonso H.W. Ngan ${ }^{\S}$ \\ Department of Mechanical Engineering, The University of Hong Kong, Pokfulam Road, \\ Hong Kong, P.R. China \\ ${ }^{\S}$ Correspondence author (email: hwngan@hku.hk)
}

\begin{abstract}
The coalescence of nano-crystals during sintering is often found to result in interesting crystalline structures such as multi-fold twins, and yet the plasticity mechanism accompanying their formation is unclear. In this work, the sintering behavior of two unsupported copper nanoparticles initially at room temperature is investigated by molecular dynamics simulations under the constant-energy ensemble. The results reveal that once the two nanoparticles are brought into contact, they often go through drastic structural changes with the inter-particle grain boundary quickly eliminated, and single- and multi-fold twinning occurs frequently in the coalesced product. Whereas the formation of single twins is found to be via the more usual mechanism of emission of Shockley partials on $\{111\}$ planes, the formation of five-fold twins, however, takes place via a novel dislocation-free mechanism involving a series of shear and rigid-body rotation processes caused by elastic waves with amplitudes not corresponding to any allowable Burgers vector in the fcc lattice. Such a lattice-wave, dislocation-free twinning mechanism has never been reported before.
\end{abstract}

\section{Keywords:}

Nanoparticle; sintering; dislocation; deformation twinning; molecular dynamics 


\section{Introduction}

The sintering of nanoparticles is a crucial mechanism occurring in a number of fabrication processes. During gas phase synthesis of materials and coatings, the interaction between nanoparticles is generally considered to comprise two sequential processes (Ulrich and Subramanian 1977), namely, collision of the approaching particles to form agglomerates, and sintering of these agglomerates, usually nonspherical in shape, to form more spherical ones by solid-state diffusion. Nanocrystalline materials can be manufactured by sintering nanoparticles as building blocks (Gleiter 1989). For monolayer nanoparticles dispersed on substrates, Ostwald ripening and coalescence, i.e. the overall interparticle growth where the initial stages comprise the agglomeration of separate particles followed by subsequent sintering, contribute to dimensional changes of the particles (José-Yacamán et al. 2005). The understanding of nanoparticle sintering is therefore essential for obtaining nanoparticles or nanocrystalline materials with desired physical and chemical properties.

Several phenomenological models have been proposed for the sintering of particles (Koch and Friedlander 1990; Friedlander and Wu 1994; German 1996; Olevsky and Molinari 2000). In the neck growth model for two-particle sintering, the curvature gradient from point to point is regarded as the driving force for atomic flux. In its simplest form, the neck size ratio, i.e. the ratio of the neck diameter to particle diameter, is expressed as a power law of the isothermal sintering time and the inverse of the particle diameter, with the power exponents and other normalizing constants depending on the sintering stage (German 1996). In a linear-rate law (Koch and Friedlander 1990; Friedlander and Wu 1994), it is assumed that the coalescence rate of an agglomerated particle is directly proportional to its surface area in excess of that of the spherical state.

These phenomenological models all treat the sintering particles as continuum, i.e. their volume and surface area rather than their crystallographic structure are considered. The crystal structures of the sintered particles are mostly not described in these models, and whether grain boundaries, twins, stacking faults and dislocations exist in the sintered particles is unclear, and the crystal orientations of the sintered particles in relation to the initial ones are not considered. 
On the other hand, several experimental observations have elucidated that the sintered nanoparticles are often far from a simple combination of the initial particles with a grain boundary in the neck region. Dai et al. (2001) found the existence of twin boundaries but the absence of grain boundaries in coalesced FePt nanocrystals. Ng and Ngan (2002) performed in situ annealing experiments in the transmission electron microscope (TEM) and observed the realtime coalescence of $\mathrm{Ni}-\mathrm{Al}$ nanograins with complete removal of the grain boundaries in between. José-Yacamán et al. (2005) detected two very small Pd nanoparticles coalescing into a single fcc crystal using high-resolution TEM (HRTEM), but they also showed that a grain boundary still remained after the coalescence between two $11 \mathrm{~nm}$ bimetallic Au-Pd nanoparticles. Single twinning and multiple twinning are observed under HRTEM in the coalescence of FePt, $\mathrm{Si}, \mathrm{Au}$ and CoPt nanocrystals (Wang et al. 2004; Wang et al. 2005; Penuelas et al. 2008; Wang et al. 2009). The crystal structural change during sintering is worth investigating, because defects such as grain boundaries, twins and dislocations in general can affect mechanical and electrical properties of the sintered particles. Also analyzing the crystal structure can provide extra insights into the nanoparticle sintering mechanism.

In contrast to the phenomenological models described above, molecular dynamics (MD) simulations do not require a priori knowledge of the responsible mechanism as input, and any crystallographic structures of the particles are also directly considered. MD simulations have therefore been used in a number of reports on the sintering between two homogeneous nanoparticles (Zhu and Averback 1996; Lewis et al. 1997; Raut et al. 1998; Zeng et al. 1998; Lehtinen and Zachariah 2001; Hendy et al. 2003; Liu et al. 2003; Arcidiacono et al. 2004; Ding et al. 2004; Hawa and Zachariah 2006; Koparde and Cummings 2008; Karkin et al. 2008; Pan et al. 2008; Kart et al. 2009; Ding et al. 2009; Karkin et al. 2010; Song and Wen 2010; Moitra et al. 2010; Antúnez-García et al. 2011). In these studies, two adjacent and unsupported nanoparticles were equilibrated under a certain condition for investigation. However, the crystallographic detail of the sintered nanoparticles has not been thoroughly investigated. Here we highlight several reports that are most relevant to this issue. Zhu and Averback (1996) found two misoriented $\mathrm{Cu}$ nanoparticles ending up with a low-energy boundary in between, while two aligned nanoparticles became a single fcc crystal after rapid sintering lasting for $100 \mathrm{ps}$ at $700 \mathrm{~K}$. Pan et al. (2008) showed the slip mechanism during initial neck growth at a low temperature. Ding et al. (2009) concluded that the different types of necks between particles (either with or 
without a grain-boundary) lead to different mechanisms of mass redistribution. Karkin et al. (2010) discovered the formation of twins and, in particular, fivefold ones, during the coalescence of Ni nanoparticles at temperature $T=780-1350 \mathrm{~K}$. Antúnez-García et al. (2011) performed simulations on the collision of small gold clusters and found that two small clusters can merge into a larger one with a regular crystal structure by reorganization. Despite these studies, the knowledge base on the change of crystal structure during nanoparticle sintering is far from systematic and comprehensive, and in particular, issues that are poorly understood include: (i) the description of possible outcomes and their probability of occurrence, (ii) the influence from initial conditions (e.g. temperature, crystal orientation, particle shape and size), and (iii) the governing mechanism.

In the present work, we investigate the sintering process of two adjacent and unsupported copper nanoparticles initially at room temperature using MD simulations under the constantenergy ensemble. Our emphasis is to identify and characterize the crystal configurations of the sintered nanoparticles. Indeed, we found drastic change of crystal structure of nanoparticles during the sintering. We performed simulations with particles of different shapes, sizes and crystal orientations, and by analyzing both the coalesced products and the evolution history, we attempt to characterize the different outcomes under various conditions and determine the governing mechanisms.

\section{Simulation method}

The MD program employed made use of the leap-frog formulation with a time step of 6 fs. Trial runs showed that the simulation results by using this time step are convergent with those obtained using a shorter time step of 1 fs. Atoms were allowed to interact through an EAM potential for $\mathrm{Cu}$ developed by Sheng et al. (2011). This potential was found to provide reasonable estimates of the surface energies, as well as stacking fault and twin energies, for $\mathrm{Cu}$ (Sheng at al. (2011)). Prior to the sintering simulations, each generated fcc copper nanoparticles was equilibrated under a constant temperature of $300 \mathrm{~K}$ for $0.6 \mathrm{~ns}(100,000$ time steps), and none of the particles underwent structural transition during the equilibration. During the subsequent sintering simulations, two individually relaxed fcc single copper nanoparticles were allowed to 
interact for $1.2 \mathrm{~ns}(200,000$ time steps) under the constant-energy ensemble starting from $T=$ 300K. Snapshots of the atomic structure were periodically stored. To facilitate the subsequent examination, after the sintering simulations, the sintered products were quenched to $0 \mathrm{~K}$ by using the conjugate gradient minimization technique.

A key aspect to study crystal structures is to locate any grain boundaries in them. To do this, the coordination number $(\mathrm{CN})$, i.e. the number of nearest neighbors, of each atom was checked, and if an atom has $\mathrm{CN}$ exactly equal to 12 within a cut-off distance of $1.05 \times a_{0} / \sqrt{2}$, we label it as an atom within a single fcc crystal, while noting that this does not rule out the atom to be on a twin boundary or a stacking fault. In a twinned crystal, the atom can be in the fcc lattice, on hcp stacking or on the symmetrical axis of a fivefold twin, and to differentiate between these cases, we used the common neighbor analysis (Honeycutt 1987).

Besides the analytical methods described above, we also inspected the crystal structures graphically by plotting the positions of the atoms in a 3-D space. Another approach used was to calculate sections of the reciprocal lattice of the atomic structure.

\section{Results}

\subsection{Overview of the simulated configurations and results}

The present work focuses on the sintering behavior between two unsupported fcc single copper nanoparticles initially separated by a small displacement. In addition to temperature, the final structure of the sintered particles may depend on the following degrees of freedom:

(i) the shapes and sizes of the two initial nanoparticles,

(ii) the orientations of the initial crystals,

(iii) the initial velocities and the separation of the two particles (e.g. the gap in between), and

(iv) the atomic disregistry factor taking into account the atomic scale displacements within crystalline periodicity of the two crystals with respect to their mathematical centers. 
In an MD study like the present work, the task to exhaust all the degrees of freedom above is a formidable one and hence the scope has to be limited somehow. For this reason, we restricted the initial shape of the two fcc nanoparticles to be either cubic or spherical, although nanoparticles can take various other morphologies (Marks 1994; Henry 2005). The number of atoms in each particle ranged from about 650 to 3000 ( $2 \mathrm{~nm}<$ particle diameter $<4 \mathrm{~nm})$. The system was constructed in the following fashion: 1) two identical fcc crystals were generated with their [100], [010] and [001] direction parallel to $x, y$ and $z$ axes, respectively, 2) one of the particles was rotated around the $z$ axis by $0,15,30$ or $45^{\circ}$, and 3 ) the centers of the two particles were placed along the $x$ axis with no initial velocity. The gap in between was always set to be $4 \AA$, and the atomic site at the center of each particle was set to coincide with the $x$ axis. A starting temperature of $300 \mathrm{~K}$ was chosen to slow down atomic diffusion and to avoid the melting of crystals. The condition of constant-energy ensemble was applied based on the assumption that the nanoparticles were freely floating in vacuum.

After allowing the two particles to interact under the assumed atomic potentials for $1.2 \mathrm{~ns}$, we analyzed the crystal structure of the sintered particles and mid-products. Within all the simulated cases in the present work, two unsupported spherical or cubic copper nanoparticles $(2 \mathrm{~nm}<$ particle diameter $<4 \mathrm{~nm}$ ) were often found to go through drastic structural changes. The grain boundary between the two initial particles often annihilates during sintering, and single and multiple twinning often exists in the sintered nanoparticle.

The subsequent part of this section is organized as follows. Section 3.2 describes the crystal structures of the sintered nanoparticles in our simulations, with particular emphasis on the twinning configurations. The effect of particle shape, size and orientation is discussed in Section 3.3. We also analyzed the reaction rime for the structural transition to occur. Section 3.4 characterizes the major processes during the crystal structural change. Section 3.5 discusses the specific mechanisms for twinning.

\subsection{The crystal structures of sintered nanoparticles}

The sintered nanoparticles adopt different crystal structures. In the total of 40 simulation cases in the present work, the crystal structures of the sintered particles can be divided into 
several categories as shown in Fig. 1. Fig. 1(a) shows an fcc crystal at the end of the sintering. Since the reciprocal lattice of the fcc lattice is bcc, the reciprocal lattice of fcc crystal viewed from the [110] lattice direction appears as a rectangular lattice as illustrated in the right panel of Fig. 1(a). Among the 40 simulation cases in the present work, single fcc crystals (case (a)) appeared 4 times.

Fig.1 (b) shows another sintered product comprising two misoriented fcc crystals with a grain boundary in between. The reciprocal lattice section on the right panel is the superposition of two lattices from each crystal, and the grid lines shown single out one lattice when viewed along its [110] direction. The structure of the sintered products in this case (case (b)) can be viewed as the simple adhesion between two initial particles without significant structural change. In 11 cases out of 40, this was the final outcome.

Fig. 1(c) illustrates the third type of sintered product which is a grain boundary-free crystal containing single twinning, the boundaries of which are marked as red lines in the left panel. The sintered resultants of this type usually have two parallel twin boundaries. The two sets of reciprocal lattices can also be viewed as mirror images about a plane orthogonal to the twin boundary. Since an intrinsic stacking fault is geometrically equivalent to a single twin with the thickness of one atomic layer spacing, we also characterize single crystals with stacking faults in the same category as single twinning. The sintered products with single twinning (case (c)) occurred in 7 simulation cases, among which the crystal had an intrinsic stacking fault in two cases.

A fivefold twin in a sintered nanoparticle is evident from Fig. 1(d). This structure consists of five crystals which are twin related to one another, with five twin boundaries intersecting on a common [110] axis. The fivefold symmetry is also revealed from the reciprocal lattice section on the right panel, where five mirror planes orthogonal to the twin planes are shown. Each of the five crystals are bounded by two $\{111\}$ planes which are also the twin planes, but in this fivefold twinned structure, the apex angle of each of the five crystals is $360^{\circ} / 5=72^{\circ}$, whereas the angle between two intersecting $\{111\}$ in the perfect fcc lattice is only $70.53^{\circ}$. This means that the five crystals in this structure are elastically strained from the perfect fcc state. This fivefold twinned configuration (case (d)) appeared just once among the 40 simulations. 
A threefold twinned structure is shown in Fig. 1(e). Here, only two twin boundaries meeting at a [110] axis can be identified, and as revealed by the reciprocal lattice pattern on the right, this structure consists of three crystalline parts, instead of five as in Fig. 1(d). Two adjacent pairs of crystalline parts are twin related to each other with a twin boundary in between, and the other pair in the lower part of the left panel is not twin related and the boundary in between is a short relaxed coincident-site-lattice grain boundary. The remaining grain boundary is often as short as a few atomic spacing. After classifying the atoms in the crystal according to which initial particle they come from, the remaining grain boundary is found to coincide with a small section of the initial inter-particle gap, and each twin boundary in the sintered crystal is located within one of the initial particles. In 6 simulation cases the sintered particles exhibited this threefold twinned structure (case (e)).

Fig. 1(f) shows a variant of the fivefold twinned structure, consisting of two fivefold twins with different orientations, each arising within one of the initial particles. The two fivefold twin structures share two common crystalline parts, while the remaining three crystalline parts are not shared (see Fig. 2(b)). The two symmetrical axes from each fivefold twins are $60^{\circ}$ apart, and Fig. 1(f) shows the view along the axis of the structure on the right side. Along this viewing direction, the fivefold twin structure on the right part of the sintered particle exhibits the same appearance as that in Fig. 1(d) where the [110] lines of atoms project onto single atomic sites and the five $\{111\}$ twin boundaries project as the five red lines shown, but the other fivefold twin structure on the left part of the particle is less obvious. The reciprocal lattice pattern on the right panel shows the fivefold twin relation similar with that in Fig. 1(d), with a set of extra dots corresponding to the other fivefold twin. The dual fivefold twinned structure (case (f)) appeared 9 times in the simulation resultants.

Besides the six typical crystal structures shown in Fig. 1, a quasi-dual fivefold twin (case (g)) and triple fivefold twin structure (case (h)) also emerged once each in the simulation resultants. The quasi-dual fivefold twin structure has one fivefold twin and one threefold twin, and the triple fivefold twin contains three fivefold twins, which is one more comparing with the dual fivefold twin structure shown in Fig. 1(f). These are not shown in Fig. 1 in the interest of space. 
The conditions under which the different sintered products occur will be described in the next section. Here, we note the common occurrence of the different variants of the fivefold twin structure. Fivefold twins are widely observed in nanosized crystals (Wang et al. 2012), see Hofmeister (1998), Gryaznov et al. (1999), and Hofmeister (2004) for extensive reviews. Ino (1966) proposed a scheme to illustrate the structures of multiple twins by arranging twin-related tetrahedra packed along $\{111\}$ faces. Fig. 2 is an illustration of the fivefold twin (Fig. 2(a)) and the dual fivefold twin (Fig. 2(b)) structures corresponding to case (d) and case (f) respectively. Also shown in the lower panels of Fig. 2 are the schematics according to the Ino method.

Besides twinning, dislocations were found to be present in 5 sintered single crystals among the 40 cases simulated. In Section 3.4, relative displacement plots are used to illustrate such dislocations.

\subsection{Reaction time and initial conditions}

We are interested in how fast the structural change of the nanoparticles takes place during sintering. The reaction time is defined as the time span for the nanoparticles to go through structural transformation and adopt one of the particular crystal structures specified in Section 3.2. For case (b), Fig. 1(b), the structural change is not significant and therefore the reaction time cannot be unambiguously identified, but for all the other cases, there exists a time instant at which the crystal structure of the sintering particles just becomes stabilized and ceases changing. One of the means to determine such a reaction time is to examine the snapshots at different time instants. Within the reaction time, the snapshots usually show tremendous variations and are noticeably different from the final sintered product. After the reaction time, however, the crystal structure by and large remains identical thereafter, except that a few surface atoms may change their positions within a small range. Another way to obtain the reaction time is by exploiting the temperature variation during sintering. As a constant-energy ensemble is used throughout the simulations, temperature is allowed to rise as the potential energy is released when two

nanoparticles bond together. It is found that the temperature change of the system is closely related to the crystallinity of the nanoparticles. Here we use the case of cubic initial particles each with 666 atoms misoriented by $45^{\circ}$ as an illustration. The evolution of the temperature and crystallinity of the structure is plotted in Fig. 3. The sintering process can be divided into three stages: Stage 1 starts from the beginning of the sintering and lasts for $6 \mathrm{ps}$, during which the 
temperature rises drastically and the fraction of the crystalline regions shrinks to a minimum value. The two initial particles are attracted to one another until they bombard and release surface energy, which causes the rapid temperature rise during this stage. Stage 2 spans from 6 ps to $57 \mathrm{ps,}$ where the temperature increases at a slower rate comparing with stage 1, and crystallinity restores to the maximum level. The rises in temperature and crystallinity are almost simultaneous - temperature rise is retarded if crystallinity increment is stalled at each time interval. As the structure becomes crystalline, potential energy is released and is converted to the kinetic energy of the atoms. The reaction time is marked by the end of stage 2. Stage 3 begins from 57 ps onwards, where the crystal structure has ceased transforming, and so both the temperature and crystallinity are stable in this stage. The temperature rise in our simulations is usually around $50 \mathrm{~K}-300 \mathrm{~K}$. For larger initial particles, the rise in temperature is smaller.

We obtained the reaction times for the sintering simulations from the two methods above, and usually, the two methods yield results within less than 10 ps. Along with the reaction times, the sintered crystal structures under different conditions are summarized in Tables 1 and 2 for spherical and cubic initial particle shapes respectively.

From Tables 1 and 2, it can be seen that the probability for a major crystal structural change to occur is quite high for both initial spherical and cubic nanoparticles smaller than $\sim 4$ $\mathrm{nm}$. The reaction time is typically on the order of tens to hundreds of picoseconds. The data vaguely reveals some connections between the final crystal configurations and initial conditions: (i) it is more likely for initial particles with smaller sizes and misorientations to undergo transition, (ii) the transformation rate is slightly higher for cubic particles than for spherical ones, and (iii) single twinning (case c) is quite likely for spherical particles while for cubic particles multiple twinning (cases $\mathrm{d}$ to $\mathrm{h}$ ) is more frequent. However, Tables 1 and 2 do not seem to reveal any systematic trend between the initial conditions and final crystallographic configurations, or the reaction time.

\subsection{Mechanisms of crystal structural change}

To reveal the mechanism for the structural changes during the sintering simulations, relative displacement plots are used to identify how nearest-neighbor atoms in the initial particles 
are displaced relative to one another during the sintering process. Fig. 4 shows the relative displacement plots of typical sintered products. Here, the atoms in two adjacent $\{110\}$ planes in the center of one of the initial particles before sintering are plotted in blue color, and the horizontal and vertical axes with respect to the lattice direction of initial nanocrystal are marked in each plot. Note that the initial crystals under analysis have their [100], [010] and [001] lattice direction parallel to $x, y$ and $z$ axes of the simulation system at the beginning of sintering, respectively. For each pair $(i, j)$ of nearest-neighbor atoms in the plot, their relative displacement $r_{i j}$ is the displacement of atom $i$ relative to that of atom $j$, and to distinguish the direction of $r_{i j}$, we define atom $i$ to be always on the right hand side of atom $j$, or when they have the same horizontal coordination, atom $i$ to be above atom $j$. In Fig. 4, the in-plane component of $r_{i j}$ is represented in both magnitude and direction by a black arrow drawn between each atomic pair, and the out-of-plane component of $r_{\mathrm{i} j}$ is represented by a vertical red arrow that points upward if the out-of-plane component of $r_{i j}$ is out of the paper and downward if into the paper. All cases shown in Fig. 4 are quenched states after the end of the simulation.

Fig. 4(a) shows a case where the sintered product is a single fcc crystal in case (a), and, as mentioned above, only atoms in one of the two initial particles, i.e. one half of the sintered product, are shown. In this case, both particle rigid-body rotation and dislocation slip are noted, and in Fig. 4(b), the rotation is subtracted from the overall relative displacements in Fig. 4(a) to make the dislocation slip events more visible. The slip events shown in Fig. 4(b) are produced by a screw dislocation with Burgers vector $\frac{1}{2}[101]$ which has cross-slipped from a (111) plane onto

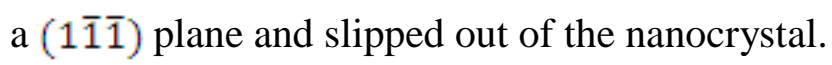

For the twinned sintered products, each successive $\{1 \overline{1} 1\}$ planes in the twinned region is displaced by a shear vector identical to the Shockley partial Burgers vector $\frac{1}{6}\langle\overline{2} \overline{1} 1\rangle$. In this case, all the Shockley partials on each slip plane are the same. In a few circumstances, we observed the slip of partials with different Burgers vectors. Fig. 4(c) shows the final relative displacements corresponding to the formation of a single twin in case (c), and the case of threefold multiple twin in case (e) is also similar. When the newly formed twin boundaries in the two sintering nanoparticles are not parallel to each other, the resultant is a threefold twin. Fig. 4(d) shows the 
relative displacement map after a fivefold twin in case (f) has been formed. The events involved are quite complicated and will be discussed separately in Section 3.5 together with those involved in the single twin formation.

Irrespective of the sintered products in the present simulation, Fig. 4 reveals that, except for a few atoms on the edges or corners of the nanoparticles, the atoms rearrange during the sintering process in a highly organized way, with no long-range relative movements of neighboring atoms. Hence, even without going into the details of the evolution history, the governing mechanism for the sintering and crystal structural changes in our simulations can be narrowed down from what has been proposed in the literature. For micron-sized particles, a quite comprehensive body of theories for solid-state sintering has been developed, and six different mechanisms are believed to be contributing, namely, surface diffusion, lattice diffusion, vapor transport, grain boundary diffusion, viscous flow, and dislocation plasticity (German 1996). It is also believed that grain growth is absent in the initial stage of sintering (German 1996). However, large relative displacements would be expected if diffusion or viscous flow mechanisms are involved. The "dislocation plasticity" in this body of literature refers to the glide of pre-existing dislocations in the initial particles, and since the present nanoparticles are dislocation-free, this mechanism is also not directly relevant, although in Fig. 4(a,b) and other examples, we did observe new dislocations nucleated from free surfaces and glided into the particles during the sintering. Furthermore, these conventional mechanisms are not able to explain the quick elimination of the inter-particle grain boundary, and the emergence of extensive twinning in our simulations. The melting of the nanoparticles due to the surface energy release was observed in some MD works (Hendy et al. 2003; Arcidiacono et al. 2004), but again, melting would cause the relative displacement map to be much more chaotic than what is shown in Fig. 4. Besides, we also performed a number of sintering simulations under a constant-temperature ensemble at $300 \mathrm{~K}$, and the quick annihilation of the inter-particle grain boundary and twinning were still observed.

From the relative displacement maps such as those shown in Fig. 4, we were able to identify three processes, namely, particle rotation, dislocation slip and an elastic-wave induced mode of twinning, and these will be discussed in detail below. Rigid-body particle rotation was observed in a number of MD works (Zhu and Averback (1996); Arcidiacono et al. (2004); Ding 
et al. (2009)). Zhu and Averback (1996) concluded that nanoparticle sintering involves a dislocation mechanism as the high shear stresses in the neck region exceed the theoretical strength of the material. In the following section, we will examine the specific mechanisms of twinning as observed from the present MD simulations.

\subsection{The formation mechanisms of twinning}

\subsubsection{Single twinning}

Fig. 5 and Fig. 6 show snapshots of relative displacement maps at different time instants during the formation of a single twin in case (c). The notations used in the maps are the same as in Fig. 4, except that the mid-products in the sintering here are not quenched, and so thermal noises are not eliminated. The initial crystal has [100], [010] and [001] lattice direction parallel to $x, y$ and $z$ axes of the simulation system, and is on the negative $\mathrm{x}$ axis direction of two particles under sintering. In Fig. 5, two (110) planes are plotted, and at $t=2.7$ ps, two Shockley partials $\left(b_{1}=\frac{1}{6}[\overline{2} \overline{1} 1] b_{2}=\frac{1}{6}[\overline{2} \overline{1} 1]\right)$ are emitted from upper right and lower right corners, respectively, to the interior of the crystal on different slip systems. At $t=3.6 \mathrm{ps}$, a few more partials have been emitted into the system, followed by more in the next few ps. Although the Shockley partial dislocations are localized in the right part of the crystal, their emission is not sequential on adjacent $\{111\}$ planes, and they propagate very quickly in the nanocrystal. Meanwhile, a few stacking faults in the system, for instance the one trailing dislocation $b_{5}$, disappear. At time 5.4 ps, the density of partials inside the crystal reaches a high value, and afterwards, probably due to the accumulated potential energy, the structure rearranges to form a single twin. Twinning slip over a large part of the crystal is evident from Fig. 5(e).

To further illustrate the glide of Shockley partials, in Fig. 6 some selected snapshots of relative displacement plots of two (111) planes parallel to the green lines in Fig. 5(a), are shown. Two Shockley partials $\left(b_{1}, b_{4}\right)$ with the same Burgers vector $\frac{1}{6}[\overline{2} \overline{1} 1]$, one on the upper right and the other at the bottom, are emitted from the free surface at around $t=2.7 \mathrm{ps}$. From $t=3.0 \mathrm{ps}$ onwards, partials $\left(b_{2}, b_{3}\right.$ and $\left.b_{6}\right)$ gliding on intersecting planes start to produce displacements on

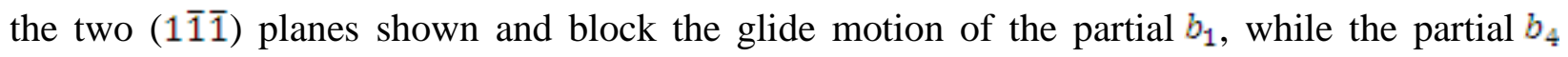


continues to glide. We calculated the propagation velocity for a number of partial dislocations and the results vary from around $2000 \mathrm{~m} / \mathrm{s}$ for the fastest one to several hundred $\mathrm{m} / \mathrm{s}$ for the slower ones.

Relative displacement maps are only capable of illustrating the distortions in a thin layer of atoms, and to provide three-dimensional visualization of the structural changes, we also plot in Fig. 7 the atoms with a large enough maximum displacement relative to their nearest neighbors for the (unquenched) mid-products. Here, the maximum relative displacement $d_{i}$ for atom $i$ is defined as:

$$
d_{i}=\max \left(\vec{r}_{i j}^{t}-\vec{r}_{i j}^{0}\right)
$$

where $j$ includes all the nearest neighbors of atom $i, \vec{r}_{i j}^{t}$ and $\vec{r}_{i j}^{0}$ are the displacement between atom $i$ and $j$ at time $t$ and at time zero when the lattice arrangements are perfect, respectively (Ngan et al. (2004)). If $d_{i}>0.7 \times a_{0} / \sqrt{6}$, then atom $i$ is marked by large blue circles in Fig. 7, and here, $a_{0} / \sqrt{6}$ is the magnitude of the Shockley Burgers vector. By this method, stacking faults, twinning as well as other defects can be identified, and from Fig. 7, the thickening and extension of such defects along close packing planes are evident. Together with the relative displacement maps in Fig. 5 and Fig. 6, it can be concluded that the defected region in the sintered nanocrystal in case (c) is indeed twinning, and this is formed by the generation and glide of Shockley partial dislocations.

\subsubsection{Fivefold twinning}

Fig. 8 shows the formation process of the fivefold twin in case (f), and only the atoms belonging to the initial crystal on the positive $\mathrm{x}$ axis direction of two particles, which has [100], [010] and [001] lattice direction parallel to $x, y$ and $z$ axes of the system before sintering simulation, are shown. In contrast to the single twin, little evidence on the gliding of Shockley partials can be found in the relative displacement maps of the unquenched mid-products during fivefold twin formation. Before time 3.6ps, a shear wave that is mainly out-of-plane in nature (i.e. along [110]) is initiated on the left surface of the crystal where impact by the other crystal is 
made, and this shear wave quickly transmits towards the other side of the crystal. Here, the shear vectors are much smaller in magnitude than the Shockley Burgers vector, which is the smaller Burgers vector allowed in the fcc lattice. After around $t=4.5 \mathrm{ps}$, in-plane displacement vectors (i.e. those normal to [110]) start to appear, and these produce two effects, (i) coupling with the initial[110] out-of-plane shear to produce twinning of the left part of the crystal, and (ii) a clockwise rotation of the upper region, and an anti-clockwise rotation of the lower region of the crystal. The in-plane shear wave and hence the rotations propagate to the right side of the crystal at a velocity of $\sim 3000 \mathrm{~m} / \mathrm{s}$ which is much higher than the $\sim 800 \mathrm{~m} / \mathrm{s}$ of the out-of-plane shear wave. For the sake of comparison, the present atomic potentials give the travelling speeds of longitudinal, out-of-plane and in-plane elastic shear waves at the long wave limit along [110] at 0K as $8200 \mathrm{~m} / \mathrm{s}, 3000 \mathrm{~m} / \mathrm{s}$ and 1680m/s, respectively (Sheng (2012); Kittel (1953)). In mere 0.6 ps, the right-most part of the nanocrystal is under the effect of in-plane vectors, and because of the opposite rotations of the upper and lower parts of the crystal, the right part of the crystal gets squeezed, and twinning occurs there as well. The fivefold twinned structure is formed and stabilized after 6ps. Throughout the whole process, the crystal structural changes are rather symmetrical about the horizontal mid-plane.

As shown in Fig. 8(f), the finally sintered crystal can be divided into five twinned parts, with a discontinuity of the relative displacement vectors on going from one part to an adjacent part. To assist the understanding of the rather complicated twining processes that lead to such a fivefold structure, Fig. 9 shows schematics to illustrate the transformation of each of the parts in Fig. 8(e). Since the structural changes are symmetric with respect to the horizontal mid-plane, only the changes in parts DAC, CAB and BAF in Fig. 8(e) are shown. As shown in Fig. 9, the crystal part $\mathrm{CAB}$ rotates clockwise as a rigid body causing the apex angle of DAC to enlarge and angle BAF to shrink. The deformation in the part DAC comprises two components: twinning and rotation. In the crystal part DAC, each successive (111) planes parallel to the eventual twin boundary AC are displaced by the same twinning vector $\frac{1}{6}[\overline{2} 11]$, although this is accomplished by first an out-of-plane shear wave mainly in the [110] direction but not of any allowed Burgers vector in the fcc lattice, followed by an in-plane shear wave, i.e. Shockley partial dislocations are not involved at all in the twinning process of DAC. Simultaneously, the later in-plane shear wave also causes part DAC to rotate clockwise so as to keep plane DA horizontal, as shown in the top 
row of schematics in Fig. 9. On the other side of $\mathrm{AC}$, part $\mathrm{CAB}$ also rotates clockwise as mentioned above, and a twin relation thus develops between DAC and CAB. Also as mentioned before, the opposite rotations of parts $\mathrm{CAB}$ and $\mathrm{EAF}$ squeeze the part BAF, and this triggers a double twinning process as shown in the bottom row of Fig. 9. Each successive (1111) planes in the crystal part BAF are displaced by a twin vector $\frac{1}{5}[112]$, and meanwhile, the successive (111) planes in the same region are displaced by the twin vector $\frac{1}{6}[11 \overline{2}]$, and again, the relative displacement snapshots in Fig. 8 indicate that Shockley partial dislocations are not involved in such a double twinning process. The combined effect of the two twinning steps corresponds to a shear of part BAF with the apex angle BAF now becoming smaller, and this accommodates the opposite rotations of parts $\mathrm{CAB}$ and EAF as mentioned above, and causes DAF to develop a twin relationship with $\mathrm{CAB}$ and $\mathrm{EAF}$.

To give further evidence for the absence of involvement of dislocations in the formation of the fivefold twinned structure, Fig. 10 shows plots of the atoms with large enough maximum displacements relative to nearest neighbors during the fivefold twin formation. Unlike the single twinning case shown in Fig. 7, the propagation of the defected regions is generally not on close packing planes, and takes place in a rather chaotic manner. In conclusion, the formation of the fivefold twinned structure takes place via a series of twinning shear and rigid-body rotation processes that are not affected by dislocation glide, but by a combination of elastic waves the amplitude of each of which does not correspond to any allowable Burgers vector in the fcc lattice.

\section{Discussions}

In the present work, we found that copper nanoparticles of $2 \mathrm{~nm}$ to $4 \mathrm{~nm}$ sizes initially at room temperature may go through major crystal structural changes during sintering coalescence. Conventional sintering mechanisms for micro-sized particles, such as the various diffusion processes, vapor transport and viscous flow (German 1996) are inconsistent with the present observed processes, which all involve short-ranged, coordinated relative displacements between neighboring atoms. Several such processes are identified in this work, including particle rotation, dislocation slip, and elastic wave-induced twinning, which are likely to be responsible for the 
plastic deformation in nanoparticles sintering during gas phase production, for example. Also, the twinning mechanism can provide an alternative explanation for the origin of the multiply twinned particles manufactured in gas-phase synthesis.

Although deformation twinning has been extensively observed in nanocrystalline materials and thin films (Hofmeister (2004); Zhu et al. (2012)), it is not generally conceived for nanoparticles beforehand. Multiple twinned particles were assumed to grow layer by layer from embryos, or form by repeated cyclic twinning (Hofmeister (2004)). The present work demonstrates that, at least theoretically, single and multiple deformation twins can emerge from nanoparticle coalescence. The simulated defected products at the end of the nanoparticle sintering are believed to be quite stable for two reasons. First, the major plasticity mechanisms during sintering (i.e. twinning, dislocation slip, etc.) are generally completed within the first hundred to a few hundred ps of sintering, and after that the crystal structures of the particles exhibit no noticeable changes until the end of the simulation up to $1.2 \mathrm{~ns}$. If these structures are unstable, some signs of changes during this time span should appear, but these are never observed in all of the 40 cases simulated. Secondly, multiply twinned particles (i.e. nanoparticles with twin defects) have been found to be naturally occurring with high yield during a number of experimental gas-phase synthesis studies, and can usually last long at room temperature (Marks 1994).

By analyzing relative displacement maps during the formation of single twins, we are able to characterize different twinning mechanisms. For single twinning, we found the emission of Shockley partial dislocations from free surfaces of the sintering particles, although this does not always take place on adjacent slip planes. When many partial dislocations are accumulated in a particular region in the nanoparticle, they intersect and cause rearrangement of the emission and glide processes to eventually produce a metastable single-twinned structure of the crystal. For fivefold twinning, the present results indicate that no dislocation mechanism is involved in the formation process. Instead, sequential emission of elastic lattice waves occurs upon impact of the two sintering particles, and this leads to a complicated chain of events involving twinning shear and rigid-body rotations, all accomplished by the elastic waves which have amplitudes not corresponding to any allowable Burgers vector in the fcc lattice. To our knowledge, this mode of twin formation has never been observed before. 
It should also be mentioned that the pole and ratchet mechanism for deformation twinning in bulk fcc materials (Christian and Mahajan 1995) is not applicable for the sintering of nanoparticles in the present work. The plasticity in nanocrystalline, yet bulk sized, $\mathrm{Cu}$ has received a lot of attention (Farrokh and Khan 2009, Barai and Weng 2009), but compared to a nano-grain inside a nanocrystalline material, the present simulated situation of two supported nano-crystals is different in terms of the surrounding constraints. For bulk sized, nanocrystalline materials, Yamakov et al. (2002) performed MD simulations and concluded that the successive emission of Shockley partials from grain boundaries onto neighboring planes is a key mechanism. Zhu et al. (2005) proposed a formation mechanism of fivefold deformation twins in nanocrystalline fcc metals, which involves different series of partial dislocations emitted from twin boundaries or grain boundaries. A single twin is formed first before it is transformed into a threefold, fourfold and fivefold twin in sequence. For copper nanoparticles in the present study, our observations are entirely different from Zhu et al.'s mechanism for nanocrystalline materials. For the single twin, the partials in our case are emitted from the free surfaces of the particles and they do not always glide on neighboring planes. For fivefold twinning, the five regions emerge rather simultaneously than sequentially, and no dislocation is involved.

The initiation of the two modes of twinning - single twinning involving Shockley dislocation generation and five-fold twinning not involving dislocations - is an interesting issue which deserves further investigation. The occurrence of both twin structures evidently depends on a chain reaction (see fig. 8 and 9) following impact, so a straight-forward comparison of the energy of the end products would provide little information on the selectivity between the two mechanisms. Whichever mechanism occurs should be the outcome of a competition between dislocation-mode and phonon-mode dispersion of the impact pressure, and to understand this, a detailed analysis of the impact dynamics needs to be carried out in the future.

Also, while the current simulations were carried out at $300 \mathrm{~K}$, the effects of higher temperatures to the coalescence process are a very interesting issue to investigate. As a first step, we also ran some simulation cases on starting at 700K. For each set of initial particle size and orientation, the higher starting temperature sometimes changes the final crystal structure of the sintered products, comparing to the results obtained at $300 \mathrm{~K}$. However, both single and multiple twinning are still extensively observed for simulations starting from $700 \mathrm{~K}$. Therefore, although 
higher temperatures may influence the sintering process, the governing twinning mechanisms may not be altered. For similar reasons, the choice of the constant-temperature (constant-NVT) ensemble, instead of the constant-energy (constant-NVE) ensemble, should also be explored. For nanoparticle sintering, very rapid heat release happens on contact due to the decrease of surface area. For instance, Fig. 3 illustrates a case where the temperature rises by about $250 \mathrm{~K}$ in a mere 60 ps. When the nanoparticles are placed on a substrate with good heat conduction, the released heat can be quickly absorbed into the heat bath. However, in gas phase production, for example, the nanoparticles are floating in the surroundings of a thin inert gas, and so heat can only be slowly dissipated through the rare collision events with the inert gas molecules. To model this scenario, the NVE ensemble was chosen to avoid the possible artifacts arising from rapid quenching in thermostat control. In some of the previous work, probably for the same considerations, the NVE ensemble was also employed to model the sintering of nanoparticles (Lehtinen and Zachariah 2001; Hendy et al. 2003). However, using the NVT ensemble should also be fruitful, especially for investigating the effects of temperature and a contacting substrate, and this should be carried out as future work.

\section{Conclusions}

Through MD simulations, we have identified and characterized the crystal structural transition processes involved in the sintering of $\mathrm{Cu}$ nanoparticles. Important findings include:

(i) Two unsupported copper nanoparticles each containing less than 3000 atoms (diameter $<4 \mathrm{~nm}$ ) are very likely to go through significant structural change during sintering. The coalesced nanoparticle may contain frequent twinning, including single, fivefold, threefold and dual fivefold twinned configurations.

(ii) The crystal structural changing process is a kinetic process largely affected by initial conditions such as particle shape, size and relative crystal orientations. The reaction time for the transformation process is usually in tens or hundreds of picoseconds. (iii) Diffusion and viscous flow are not found in our simulations. Instead, coordinated nearest-neighbor movements including dislocation slip and elastic-wave induced twinning shear, as well as rigid-body rotation, contribute to the structural changes of the 
nanoparticles. In particular, the single deformation twin is formed by the emission of Shockley partial dislocations from free surfaces, followed by some intersection and rearrangement processes. The formation of fivefold twinning does not involve dislocations, but is via a series of twinning shear and rigid-body rotation events that are accomplished by elastic waves of amplitudes not corresponding to any allowable Burgers vector in the fcc lattice.

\section{Acknowledgements}

The work described in this paper was supported by a grant from the Research Grants Council (Project No. 7159/10E) of the Hong Kong Special Administrative Region.

\section{References}

Antúnez-García J, Mejía-Rosales S, Pérez-Tijerina E, Martín J, Montejano-Carrizales, JoséYacaman M (2011) Coalescence and Collisions of Gold Nanoparticles. Materials (4):368-379. doi:10.3390/ma4020368

Arcidiacono S, Bieri NR, Poulikakos D, Grigoropoulos CP (2004) On the coalescence of gold nanoparticles. International Journal of Multiphase Flow 30 (7-8):979-994. doi:10.1016/j.ijmultiphaseflow.2004.03.006

Barai P, Weng GJ (2009) Mechanics of very fine-grained nanocrystalline materials with contributions from grain interior, GB zone, and grain-boundary sliding. International Journal of Plasticity 25: 2410-2434

Christian JW, Mahajan S (1995) Deformation twinning. Progress in Materials Science 39 (12):1-157. doi:10.1016/0079-6425(94)00007-7

Dai ZR, Sun S, Wang ZL (2001) Phase Transformation, Coalescence, and Twinning of Monodisperse FePt Nanocrystals. Nano Letters 1 (8):443-447. doi:10.1021/n10100421

Ding F, Rosén A, Bolton K (2004) Size dependence of the coalescence and melting of iron clusters: A molecular-dynamics study. Physical Review B 70 (7):075416 
Ding L, Davidchack RL, Pan J (2009) A molecular dynamics study of sintering between $\begin{array}{lllll}\text { nanoparticles. Computational Materials Science } 45 & \text { (2):247-256. }\end{array}$ doi:10.1016/j.commatsci.2008.09.021

Fang ZZ, Wang H (2008) Densification and grain growth during sintering of nanosized particles. International Materials Reviews 53 (6):326-352. doi:10.1179/174328008x353538

Farrokh B, Khan AS (2009) Grain size, strain rate, and temperature dependence of flow stress in ultra-fine grained and nanocrystalline $\mathrm{Cu}$ and $\mathrm{Al}$ : Synthesis, experiment, and constitutive modeling. Internationa Journal of Plascticity 25: 715-732

Friedlander SK, Wu MK (1994) Linear rate law for the decay of the excess surface area of a coalescing solid particle. Physical Review B 49 (5):3622-3624

German RM (1996) Sintering Theory and Practice. John Wiley \& Sons, Inc.,

Gleiter H (1989) Nanocrystalline materials. Progress in Materials Science 33 (4):223-315. doi:10.1016/0079-6425(89)90001-7

Gryaznov VG, Heydenreich J, Kaprelov AM, Nepijko SA, Romankov AE, Urban J (1999) Pentagonal Symmetry and Disclinations in small particles. Cryst Res Technol 34:10911119

Hawa T, Zachariah MR (2006) Coalescence kinetics of unequal sized nanoparticles. Journal of Aerosol Science 37 (1):1-15. doi:10.1016/j.jaerosci.2005.02.007

Hendy S, Brown SA, Hyslop M (2003) Coalescence of nanoscale metal clusters: Moleculardynamics study. Physical Review B 68 (24):241403

Henry CR (2005) Morphology of supported nanoparticles. Progress in Surface Science 80 (34):92-116. doi:10.1016/j.progsurf.2005.09.004

Hofmeister H (1998) Forty Years Study of Fivefold Twinned Structures in Small Particles and Thin Films. Crystal Research and Technology 33 (1):3-25

Hofmeister H (2004) Fivefold Twinned Nanoparticles. Encyclopedia of Nanoscience and Nanotechnology vol. 3:431-452

Honeycutt JD, Andersen HC (1987) Molecular dynamics study of melting and freezing of small Lennard-Jones clusters. The Journal of Physical Chemistry 91 (19):4950-4963. doi:10.1021/j100303a014

Ino S (1966) Epitaxial Growth of Metals on Rocksalt Faces Cleaved in Vacuum. II. Orientation and Structure of Gold Particles Formed in Ultrahigh Vacuum. J Phys Soc Jpn 21:346-362 
José-Yacamán M, Gutierrez-Wing C, Miki M, Yang DQ, Piyakis KN, Sacher E (2005) Surface Diffusion and Coalescence of Mobile Metal Nanoparticles. The Journal of Physical Chemistry B 109 (19):9703-9711. doi:10.1021/jp0509459

Karkin I, Karkina L, Gornostyrev YN (2008) Formation of Multiply-Twinned Particles during Two-Nanoparticles Agglomeration: The Results of MD Simulation. Materials Science Forum Volumes 584 - 586:1033-1038

Karkin IN, Gornostyrev YN, Karkina LE (2010) Molecular Dynamics Simulation of the Formation of Twin Boundaries during Agglomeration of Nanoparticles. Physics of the Solid State Vol. 52:431-435

Kart HH, Wang G, Karaman I, Cagin T (2009) Molecular Dynamics Study of the Coalescence of equal and unequal sized $\mathrm{Cu}$ Nanoparticles. International Journal of Modern Physics $\mathrm{C}$ Vol. 20 (2):179-196. doi:10.1142/S0129183109013534

Kittel C (1953) Introduction to solid state physics. John Wiley \& Sons

Koch W, Friedlander SK (1990) The effect of particle coalescence on the surface area of a coagulating aerosol. Journal of Colloid and Interface Science 140 (2):419-427. doi:10.1016/0021-9797(90)90362-r

Koparde V, Cummings P (2008) Sintering of titanium dioxide nanoparticles: a comparison between molecular dynamics and phenomenological modeling. Journal of Nanoparticle Research 10 (7):1169-1182. doi:10.1007/s11051-007-9342-3

Lehtinen KEJ, Zachariah MR (2001) Effect of coalescence energy release on the temporal shape evolution of nanoparticles. Physical Review B 63 (20):205402

Lewis LJ, Jensen P, Barrat J-L (1997) Melting, freezing, and coalescence of gold nanoclusters. Physical Review B 56 (4):2248-2257

Liu HB, José-Yacaman M, Perez R, Ascencio JA (2003) Studies of nanocluster coalescence at high temperature. Applied Physics A: Materials Science \& Processing 77 (1):63-67. doi:10.1007/s00339-002-2028-x

Marks LD (1994) Experimental studies of small particle structures. Reports on Progress in Physics 57 (6):603

Moitra A, Kim S, Kim S-G, Park SJ, German RM, Horstemeyer MF (2010) Investigation on sintering mechanism of nanoscale tungsten powder based on atomistic simulation. Acta Materialia 58 (11):3939-3951. doi:10.1016/j.actamat.2010.03.033 
Ng HP, Ngan AHW (2002) An in situ transmission electron microscope investigation into grain growth and ordering of sputter-deposited nanocrystalline Ni3Al thin films. J Mater Res Vol.17:2085-2094

Ngan AHW, Wen M, Woo CH (2004) Atomistic simulations of Paidar-Pope-Vitek lock formation in Ni3Al. Computational Materials Science 29 (3):259-269. doi:10.1016/j.commatsci.2003.10.003

Olevsky E, Molinari A (2000) Instability of Sintering of Porous Bodies. International Journal of Plasticity 16: 1-37

Pan H, Ko SH, P.Grigoropoulos C (2008) The Solid-State Neck Growth Mechanisms in Low Energy Laser Sintering of Gold Nanoparticles : A Molecular Dynamics Simulation Study, vol 130. vol 9. American Society of Mechanical Engineers, New York, NY, ETATSUNIS

Penn RL (2004) Kinetics of Oriented Aggregation. The Journal of Physical Chemistry B 108 (34):12707-12712. doi:10.1021/jp036490+

Penuelas J, Andreazza-Vignolle C, Andreazza P, Ouerghi A, Bouet N (2008) Temperature effect on the ordering and morphology of CoPt nanoparticles. Surface Science 602 (2):545-551. doi:10.1016/j.susc.2007.11.002

Raut JS, Bhagat RB, Fichthorn KA (1998) Sintering of aluminum nanoparticles: A molecular dynamics study. Nanostructured Materials 10 (5):837-851. doi:10.1016/s09659773(98)00120-2

Sheng HW, Kramer MJ, Cadien A, Fujita T, Chen MW (2011) Highly optimized embeddedatom-method potentials for fourteen fcc metals. Physical Review B 83 (13):134118

Song P, Wen D (2010) Molecular dynamics simulation of the sintering of metallic nanoparticles. Journal of Nanoparticle Research 12:823-829

Ulrich GD, Subramanian NS (1977) III. Coalescence as a Rate-Controlling Process. Combustion Science and Technology 17 (3-4):119-126. doi:10.1080/00102207708946822

Wang B, Idrissi H, Galceran M, Colla MS, Turner S, Hui S, Raskin JP, Pardoen T, Godet S, Schryvers D (2012) Advanced TEM investigation of the plasticity mechanisms in nanocrystalline freestanding palladium films with nanoscale twins. International Journal of Plasticity 37: 140-156 
Wang YQ, Liang WS, Geng CY (2009) Coalescence Behavior of Gold Nanoparticles. Nanoscale Research Letters 4 (7):684-688. doi:10.1007/s11671-009-9298-6

Wang YQ, Smirani R, Ross GG (2004) Nanotwinning in Silicon Nanocrystals Produced by Ion Implantation. Nano Letters 4 (10):2041-2045. doi:10.1021/n1048764q

Wang YQ, Smirani R, Ross GG, Schiettekatte F (2005) Ordered coalescence of Si nanocrystals in $\mathrm{SiO}_{-}\{2\}$. Physical Review B 71 (16):161310

Yamakov V, Wolf D, Phillpot SR, Gleiter H (2002) Deformation twinning in nanocrystalline Al by molecular-dynamics simulation. Acta Materialia 50 (20):5005-5020. doi:10.1016/s1359-6454(02)00318-X

Zeng P, Zajac S, Clapp PC, Rifkin JA (1998) Nanoparticle sintering simulations. Materials Science and Engineering: A 252 (2):301-306. doi:10.1016/s0921-5093(98)00665-0

Zhu H, Averback RS (1996) Sintering processes of two nanoparticles: A study by molecular dynamics simulations. Philosophical Magazine Letters 73 (1):27-33. doi:10.1080/095008396181073

Zhu YT, Liao XZ, Valiev RZ (2005) Formation mechanism of fivefold deformation twins in nanocrystalline face-centered-cubic metals. Applied Physics Letters 86 (10):103112103112-103113. doi:10.1063/1.1879111

Zhu YT, Liao XZ, Wu XL (2012) Deformation twinning in nanocrystalline materials. Progress in Materials Science 57 (1):1-62. doi:10.1016/j.pmatsci.2011.05.001 
Table 1: Crystal structure of sintered products and reaction time for spherical nanoparticles with different initial sizes and misorientations.

\begin{tabular}{|l|l|l|l|l|}
\hline $\begin{array}{l}\text { Number of atoms in each } \\
\text { initial particle/ diameter } D\end{array}$ & 0 degree & 15 degrees & 30 degrees & 45 degrees \\
\hline $627(D=2.4 \mathrm{~nm})$ & Case (c) in $10 \mathrm{ps}$ & Case (e*) in $300 \mathrm{ps}$ & Case (e) in $30 \mathrm{ps}$ & Case (e) in $600 \mathrm{ps}$ \\
\hline $959(D=2.8 \mathrm{~nm})$ & Case (a) in $20 \mathrm{ps}$ & Case (c) in $10 \mathrm{ps}$ & Case (c) in $50 \mathrm{ps}$ & Case (b) \\
\hline $1505(D=3.2 \mathrm{~nm})$ & Case (a) in $20 \mathrm{ps}$ & Case (b) & Case (e*) in 300 ps & Case (b) \\
\hline $2093(D=3.6 \mathrm{~nm})$ & Case (b) & Case (b) & Case (b) & Case (b) \\
\hline $3055(D=4.0 \mathrm{~nm})$ & Case (a) in $20 \mathrm{ps}$ & Case (a) in $20 \mathrm{ps}$ & Case (b) & Case (b) \\
\hline
\end{tabular}

Note: Cases (a) to (e) are according to Fig. 1(a-e) respectively. The reaction time above has a maximum error of 10 ps. "*” denotes that dislocations are in the sintered products.

Table 2: Crystal structure of sintered products and reaction time for cubic nanoparticles with different initial sizes and misorientations.

\begin{tabular}{|l|l|l|l|l|}
\hline $\begin{array}{l}\text { Number of atoms in each } \\
\text { initial particle/ diameter } D\end{array}$ & 0 degree & 15 degrees & 30 degrees & 45 degrees \\
\hline $666(D=2.5 \mathrm{~nm})$ & Case (c) in $30 \mathrm{ps}$ & Case (f) in $50 \mathrm{ps}$ & Case (f) in $120 \mathrm{ps}$ & Case (d) in $60 \mathrm{ps}$ \\
\hline $1099(D=2.6 \mathrm{~nm})$ & Case (f) in $60 \mathrm{ps}$ & Case (f) in $70 \mathrm{ps}$ & Case (f*) in $140 \mathrm{ps}$ & Case (c) in $300 \mathrm{ps}$ \\
\hline $1688(D=3.3 \mathrm{~nm})$ & Case (c) in $10 \mathrm{ps}$ & Case (f*) in $30 \mathrm{ps}$ & Case (g) in $80 \mathrm{ps}$ & Case (e) in $120 \mathrm{ps}$ \\
\hline $2048(D=3.6 \mathrm{~nm})$ & Case (f) in $30 \mathrm{ps}$ & Case (f) in $20 \mathrm{ps}$ & Case (h) in $130 \mathrm{ps}$ & Case (b) \\
\hline $2916(D=4.0 \mathrm{~nm})$ & Case (c) in $270 \mathrm{ps}$ & Case (f) in $500 \mathrm{ps}$ & Case (e*) in $200 \mathrm{ps}$ & Case (b) \\
\hline
\end{tabular}

Note: Cases (a) to (e) are according to Fig. 1(a-e) respectively. The reaction time above has a maximum error of 10 ps. "*” denotes that dislocations are in the sintered products. 


\section{Figure Captions}

Fig. 1 Crystal structure of resultants after 1.2 ns of sintering. Left: All the atoms are plotted as points in the 3-D space along the common [110] lattice direction for all or some of the crystal parts. Notice that under such a perspective, any line of atoms along [110] projects into one single atom, twin boundaries appear as lines, and fivefold symmetrical axis emerges as a point. Twin boundaries are marked by red lines. Coincident-site-lattice grain boundary is marked in case (e) by a blue line. Right: The projections of the reciprocal lattice along [110] direction. The intersection points of each set of colored grid lines denote the reciprocal lattice of one fcc crystalline fraction. Dense grey lines denote mirror planes.

Fig. 2 The sintered products with (a) fivefold twin and (b) dual fivefold twin structures corresponding to case (d) and (f) respectively. Black dots: atoms in fcc lattice or on the surface. Blue dots: atoms on a twin boundary. Blue lines: bonds unique for hcp stacking. Red dots: atoms on fivefold symmetrical axis. Red line: bonds unique for fivefold symmetrical axes. The identification was accomplished by common neighbor analysis (Honeycutt 1987). The lower panels show schematics of the structures according to the method by Ino (1966).

Fig. 3 Temperature and crystallinity evolution during sintering of two cubic initial particles each with 666 atoms misoriented by $45^{\circ}$. Crystallinity is the fraction of atoms with $12 \mathrm{CN}$. The mid-products are quenched prior to the $\mathrm{CN}$ checking analysis. Note that crystallinity can never reach unity for nanoparticles because of surface atoms.

Fig. 4 Relative displacement maps for typical sintered products after quenching at the end of the simulation. The initial conditions are specified below each plot in the form of (number of atoms in each initial particles)_(crystal misorientation).

Fig. 5 Relative displacement map of two (110) planes for the sintering of two aligned cubic initial particles each with 2916 atoms. Time is indicated below each snapshot. The left part of the crystal, in which little deformation occurs, is not drawn to save space.

Fig. 6 Relative displacement map of two (111) planes for the case shown in Fig. 5. Time is indicated below each snapshot. 
Fig. 7 Crystal structure change during the formation of single twin in the case shown in Figs. 5 and 6. Atoms with large maximum relative displacements are marked by large blue circles. Other atoms are indicated by small black dots. Only atoms belonging to the initial crystal on the negative $\mathrm{x}$ axis are drawn. The box frame indicates the approximate shape of the particle. Time is indicated below each snapshot.

Fig. 8 Relative displacement maps for sintering of two cubic initial particles each with 2048 atoms misoriented by $15^{\circ}$, to form a fivefold twinned structure as in case (f). Time is indicated below each snapshot.

Fig. 9 Schematic illustration for the formation of fivefold twin. Solid frames and dashed frames represent the shape of each part after and before each operation. Blue lines indicate twin boundaries.

Fig. 10 Crystal structure change during the formation of fivefold twin. Atoms with large maximum relative displacement are marked by large blue circle. Other atoms are indicated by small black dots. Only atoms belonging to the initial crystal on the positive $\mathrm{x}$ axis are drawn. The box frame indicates the approximate shape of the particle. Time is indicated below each snapshot. 

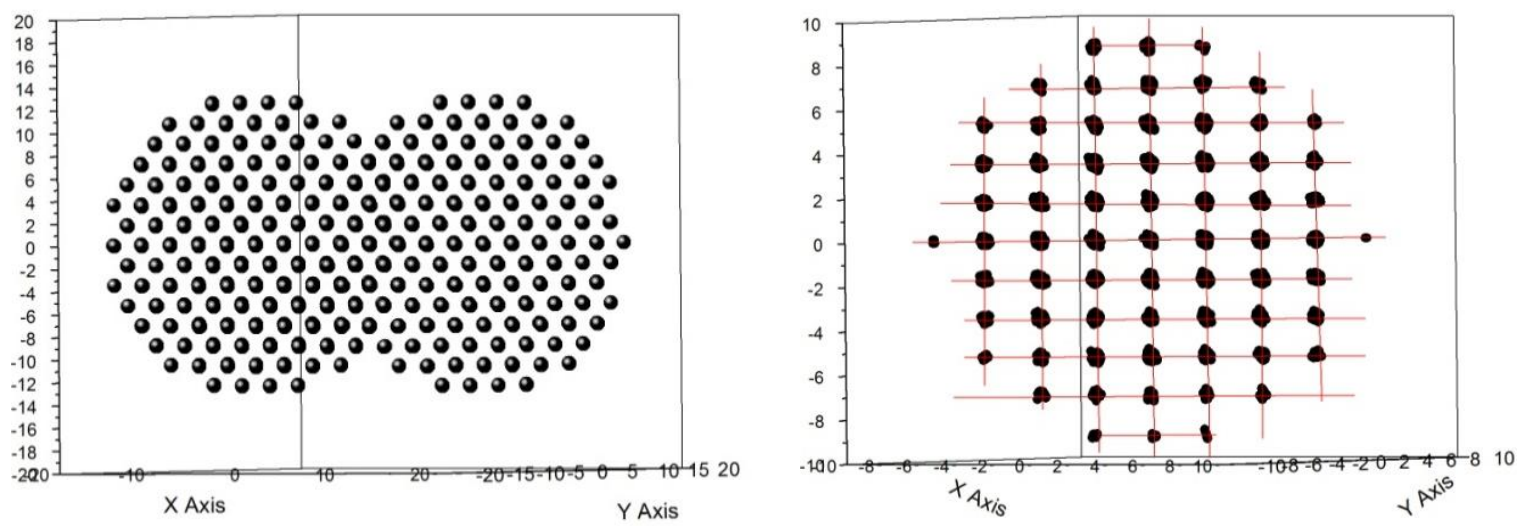

(a) fcc single crystal, case (a)
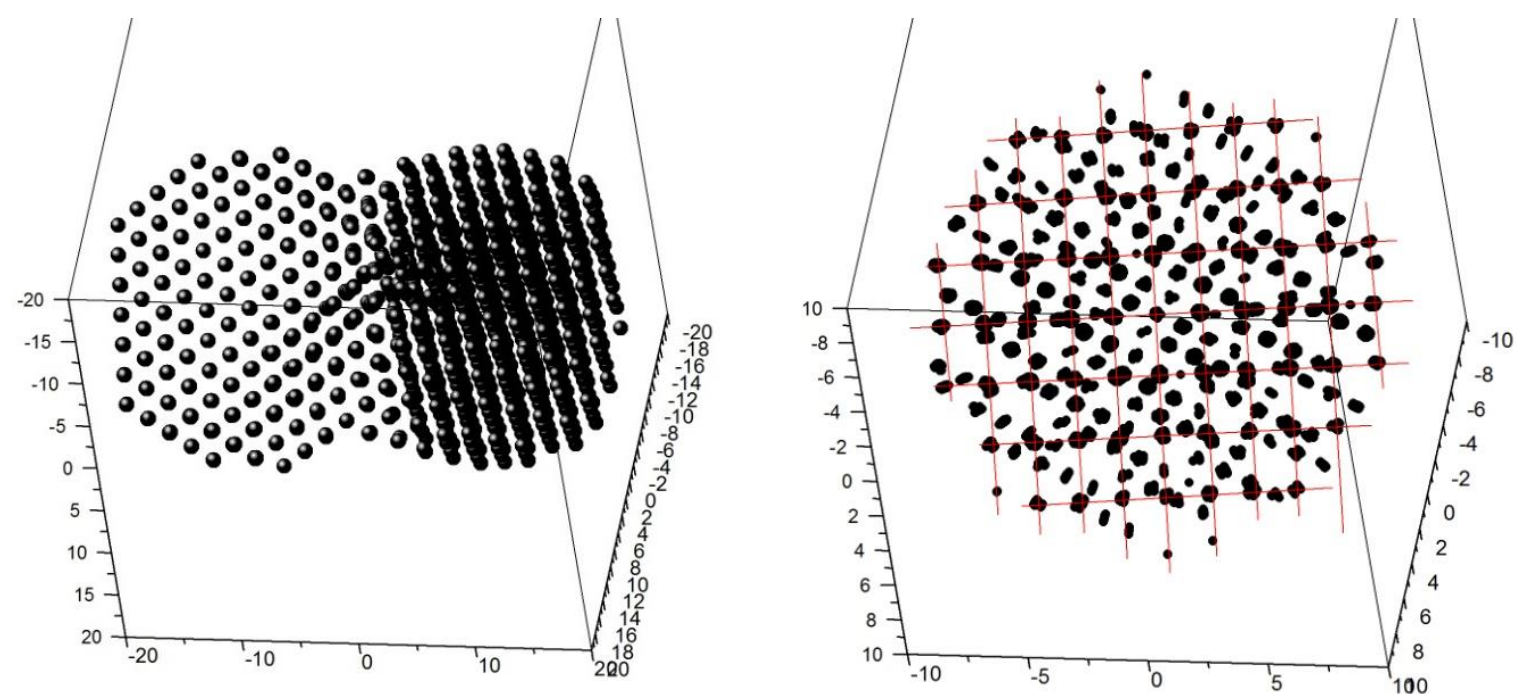

(b) Two misoriented crystals, case (b)
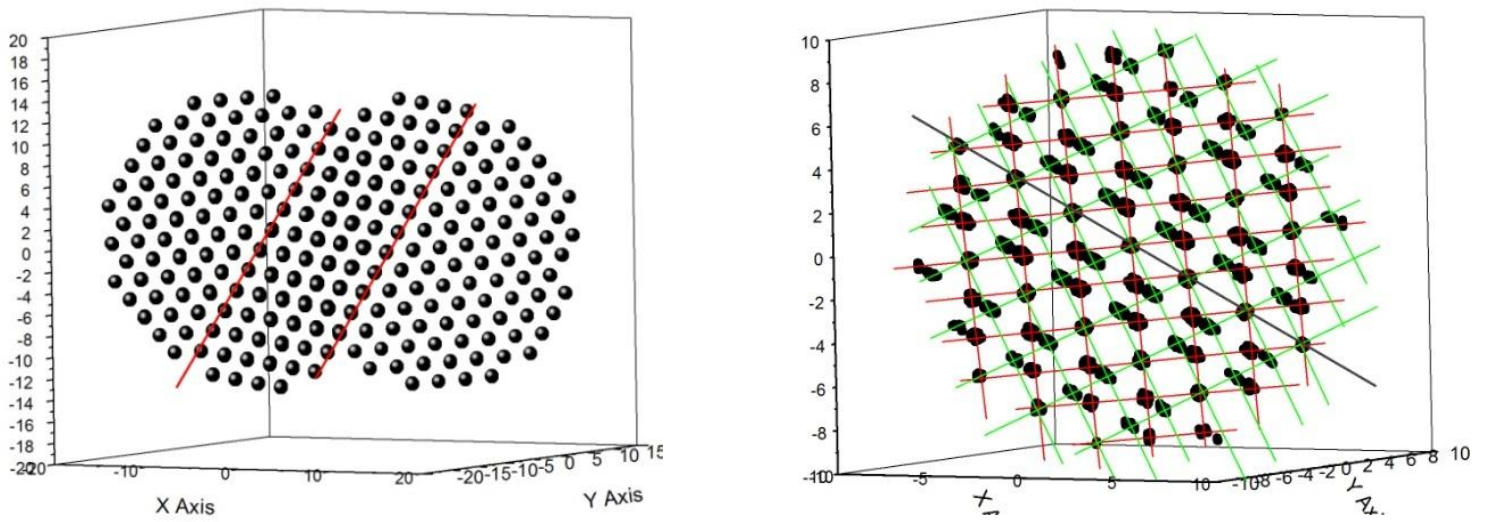

(c) Single twinning, case (c) 

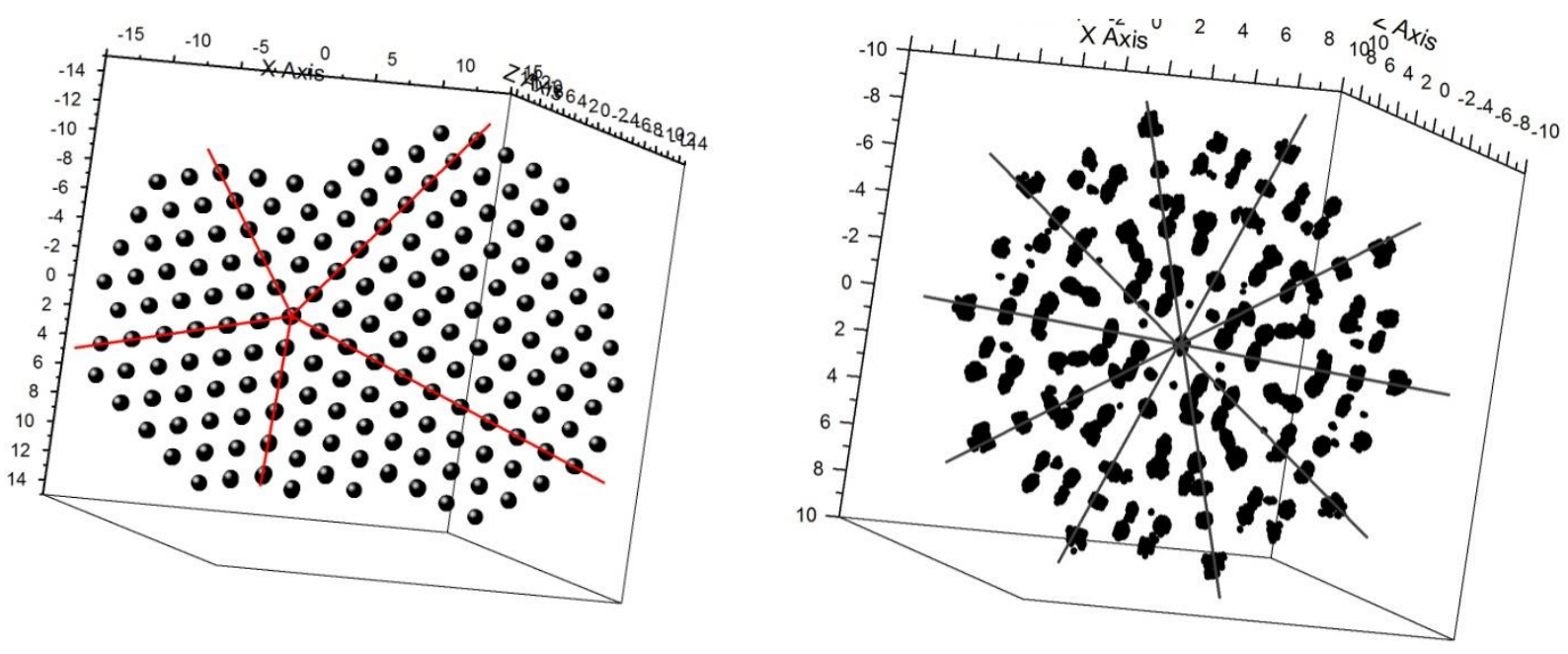

(d) fivefold twinning, case (d)
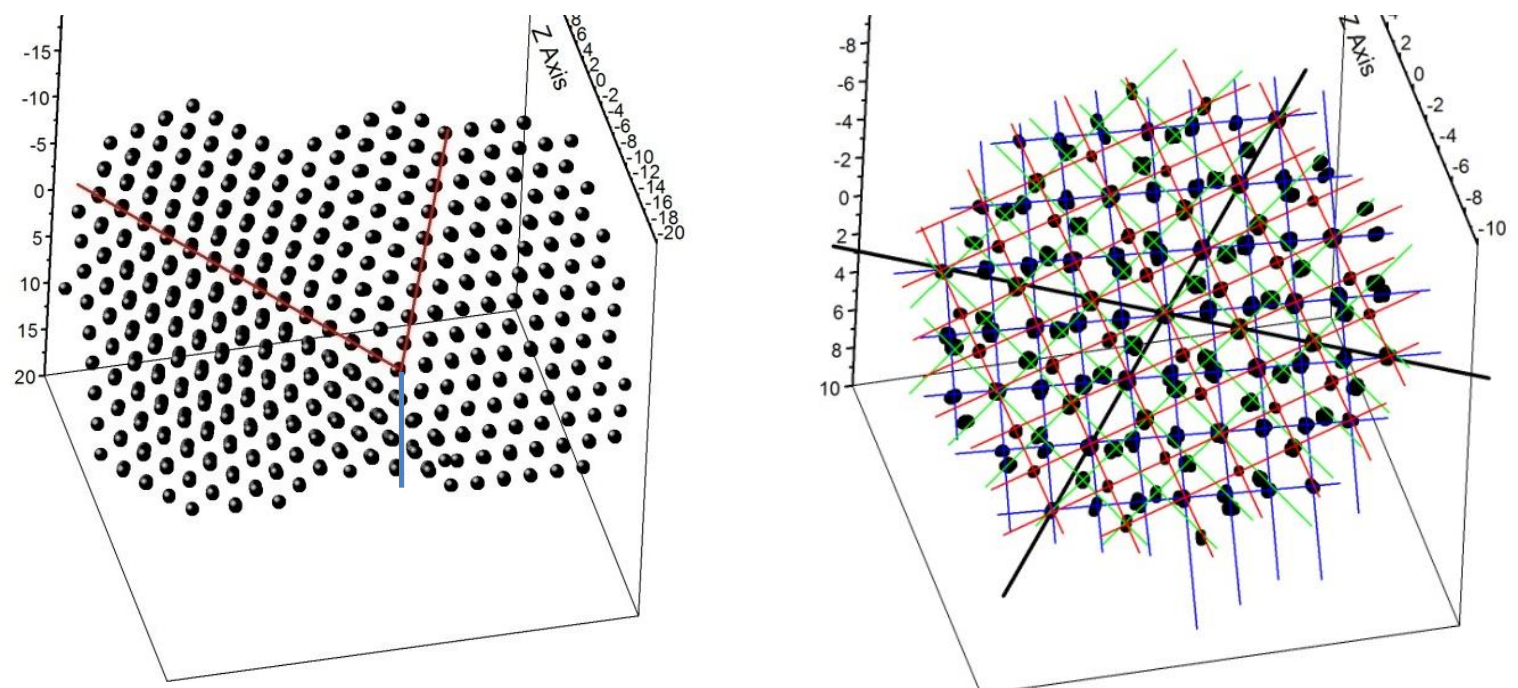

(e) threefold twinning, case (e)
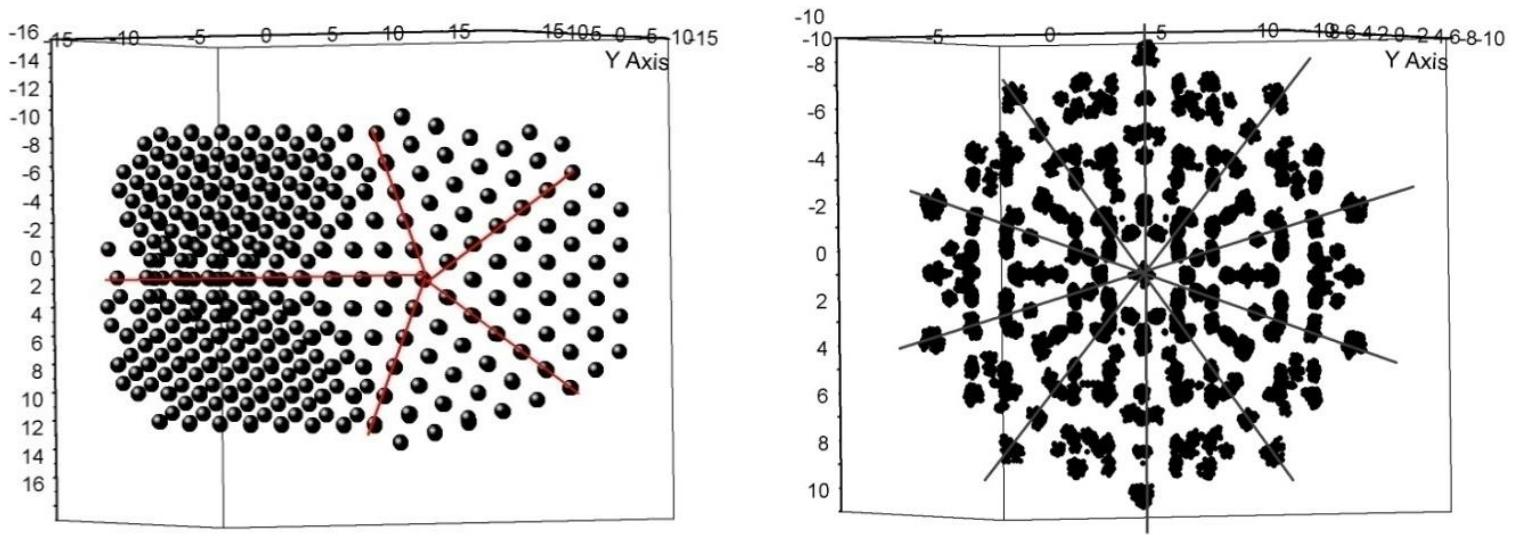

(f) dual fivefold twinning, case (f) 
Fig. 1 Crystal structure of resultants after 1.2 ns of sintering. Left: All the atoms are plotted as points in the 3-D space along the common [110] lattice direction for all or some of the crystal parts. Notice that under such a perspective, any line of atoms along [110] projects into one single atom, twin boundaries appear as lines, and fivefold symmetrical axis emerges as a point. Twin boundaries are marked by red lines. Coincident-site-lattice grain boundary is marked in case (e) by a blue line. Right: The projections of the reciprocal lattice along [110] direction. The intersection points of each set of colored grid lines denote the reciprocal lattice of one fcc crystalline fraction. Dense grey lines denote mirror planes. 

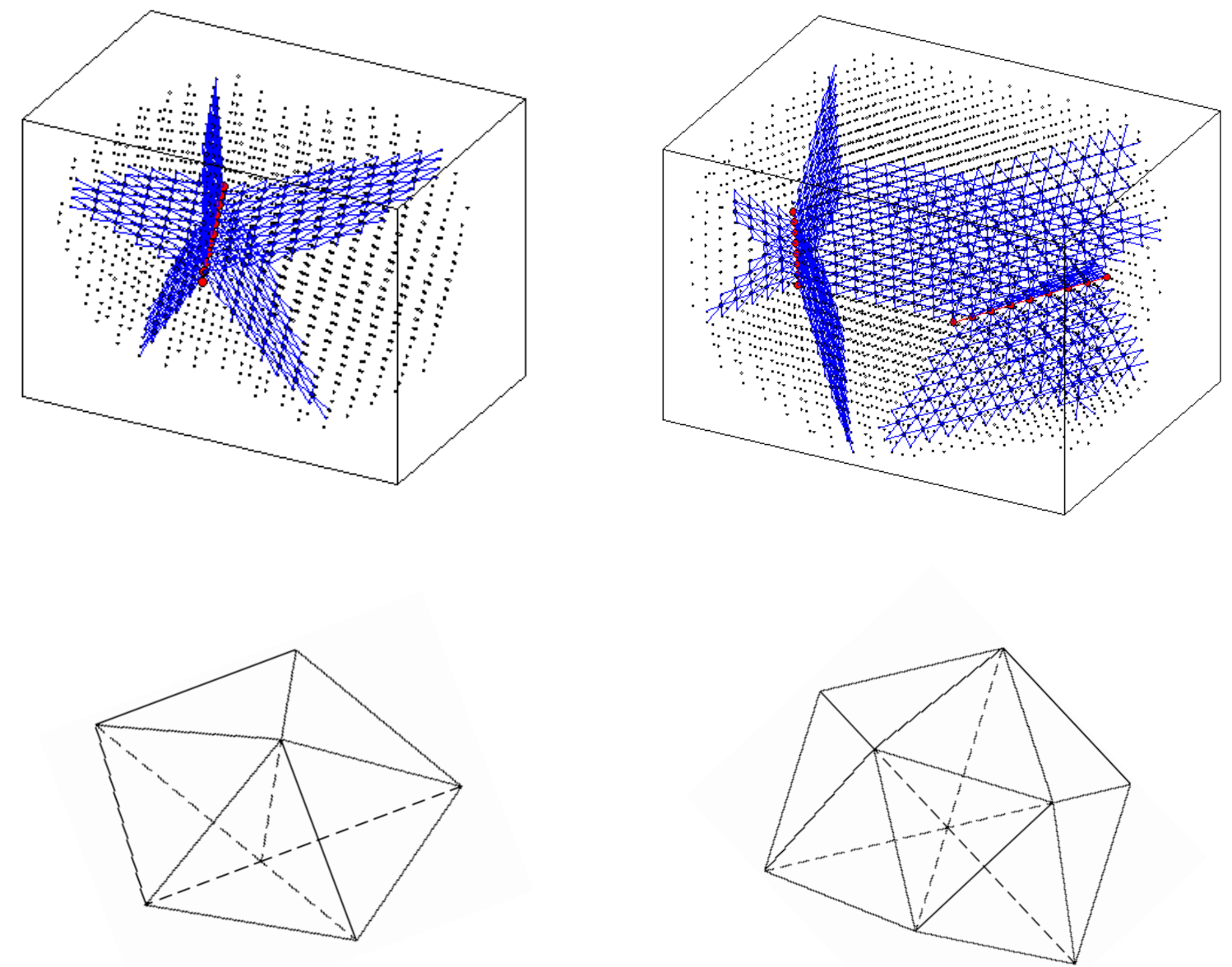

(a)

(b)

Fig. 2 The sintered products with (a) fivefold twin and (b) dual fivefold twin structures corresponding to case (d) and (f) respectively. Black dots: atoms in fcc lattice or on the surface. Blue dots: atoms on a twin boundary. Blue lines: bonds unique for hcp stacking. Red dots: atoms on fivefold symmetrical axis. Red line: bonds unique for fivefold symmetrical axes. The identification was accomplished by common neighbor analysis (Honeycutt 1987). The lower panels show schematics of the structures according to the method by Ino (1966). 


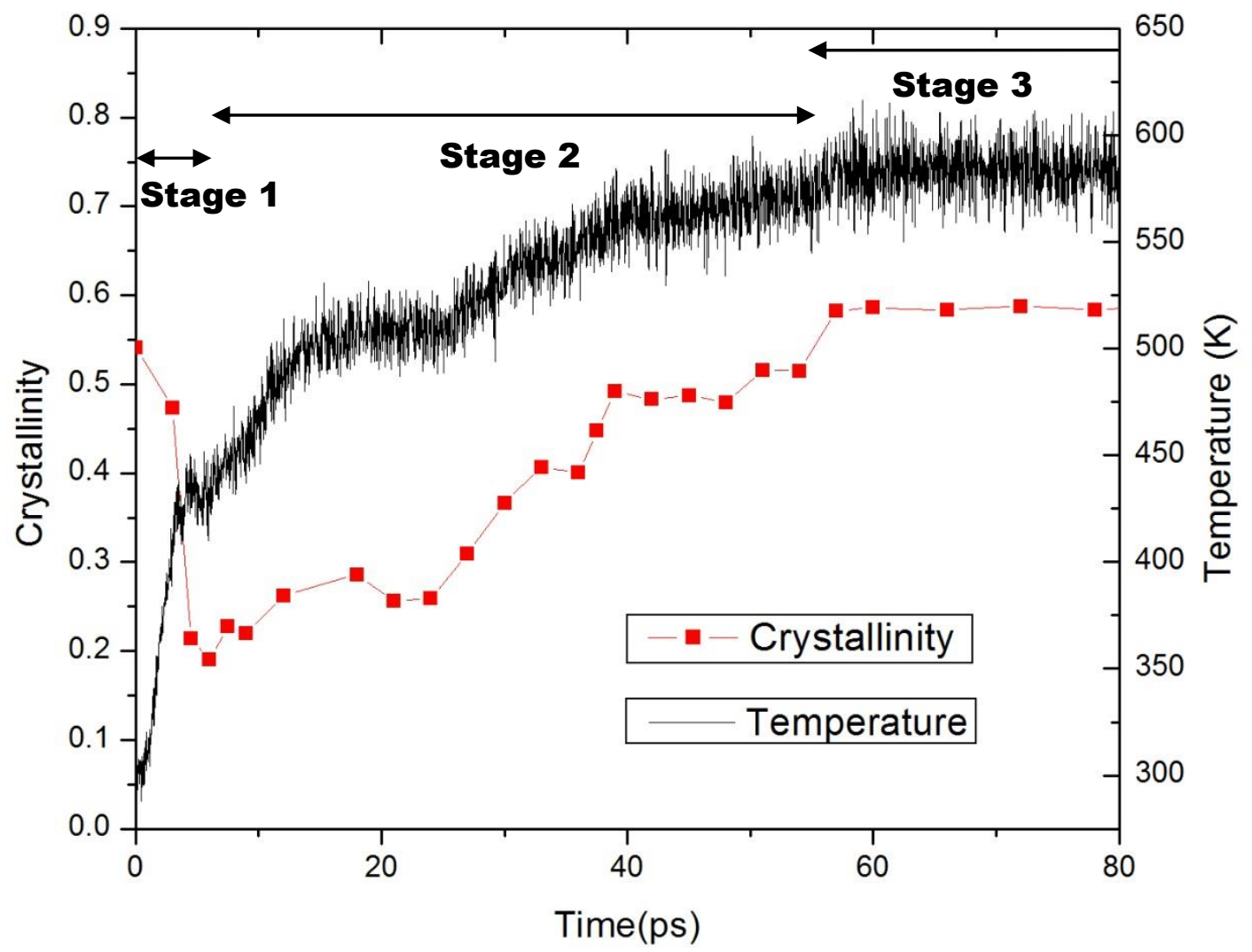

Fig. 3 Temperature and crystallinity evolution during sintering of two cubic initial particles each with 666 atoms misoriented by $45^{\circ}$. Crystallinity is the fraction of atoms with $12 \mathrm{CN}$. The mid-products are quenched prior to the $\mathrm{CN}$ checking analysis. Note that crystallinity can never reach unity for nanoparticles because of surface atoms. 


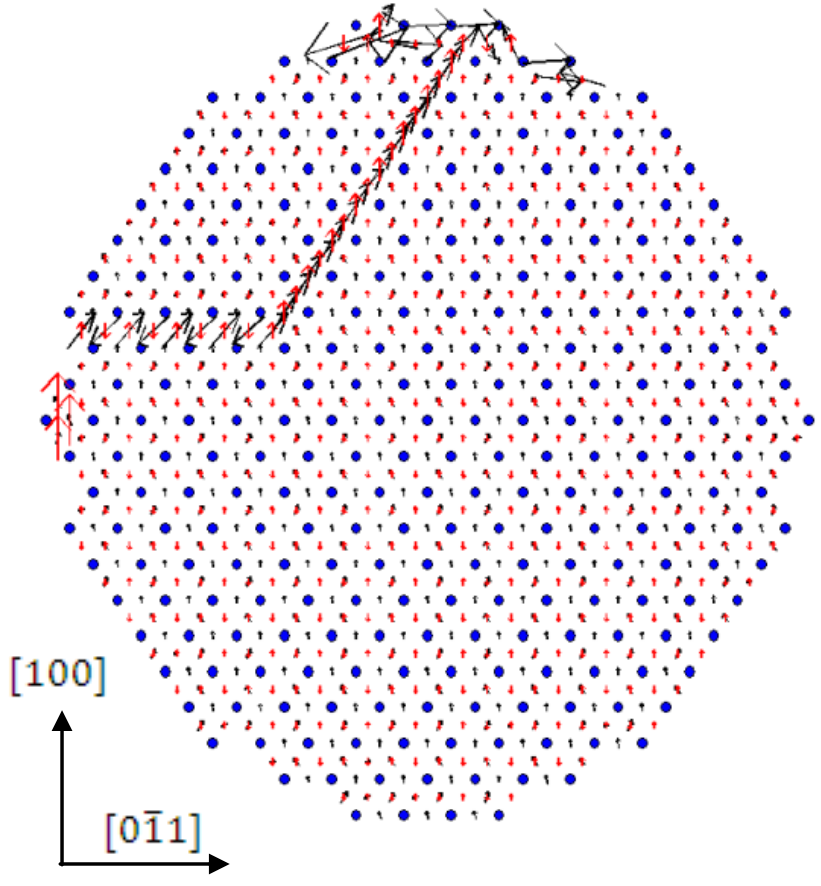

(a) 3055_15degree, case (a)

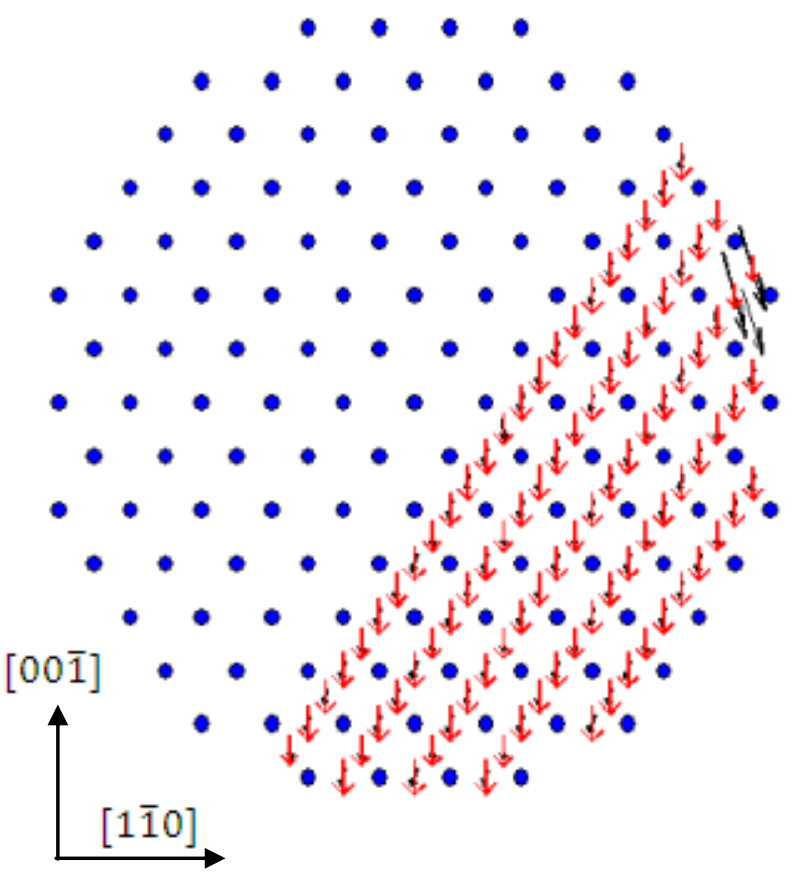

(c) 959_15degree, case (c)

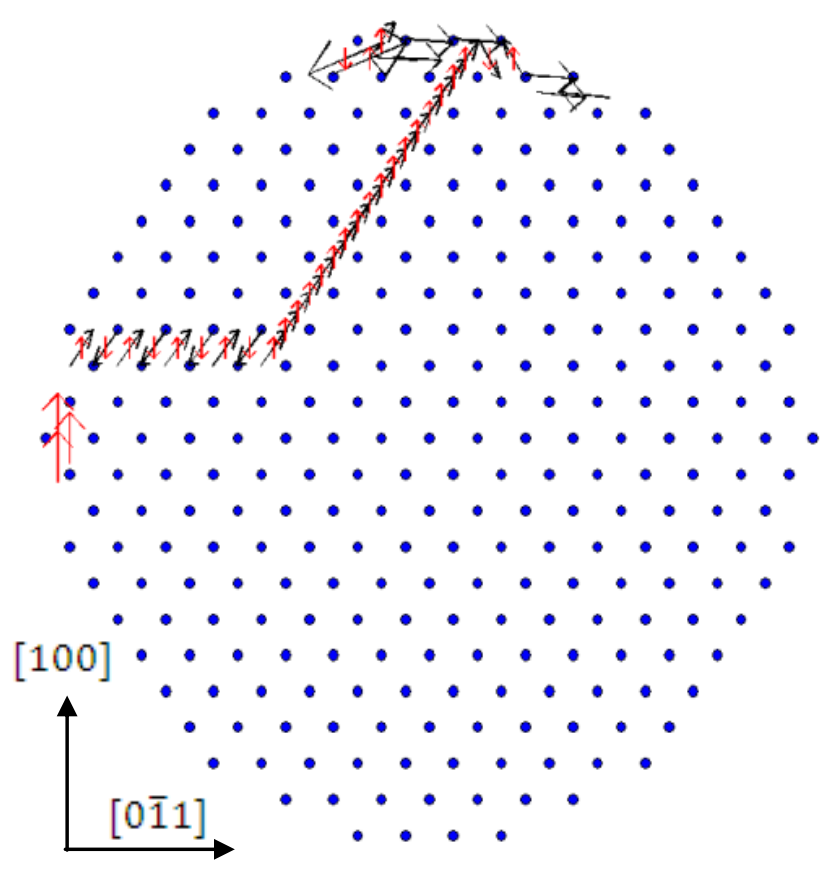

(b) 3055_15degree (rotation removed)

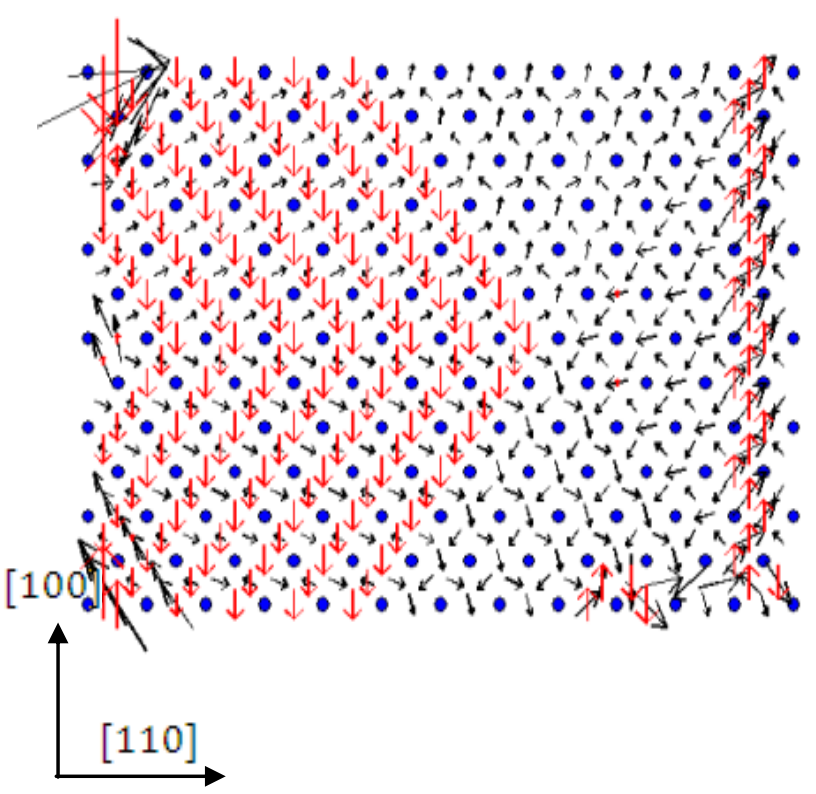

(d) 1099_15degree, case (f) 
Fig. 4 Relative displacement maps for typical sintered products after quenching at the end of the simulation. The initial conditions are specified below each plot in the form of (number of atoms in each initial particles)_(crystal misorientation). 


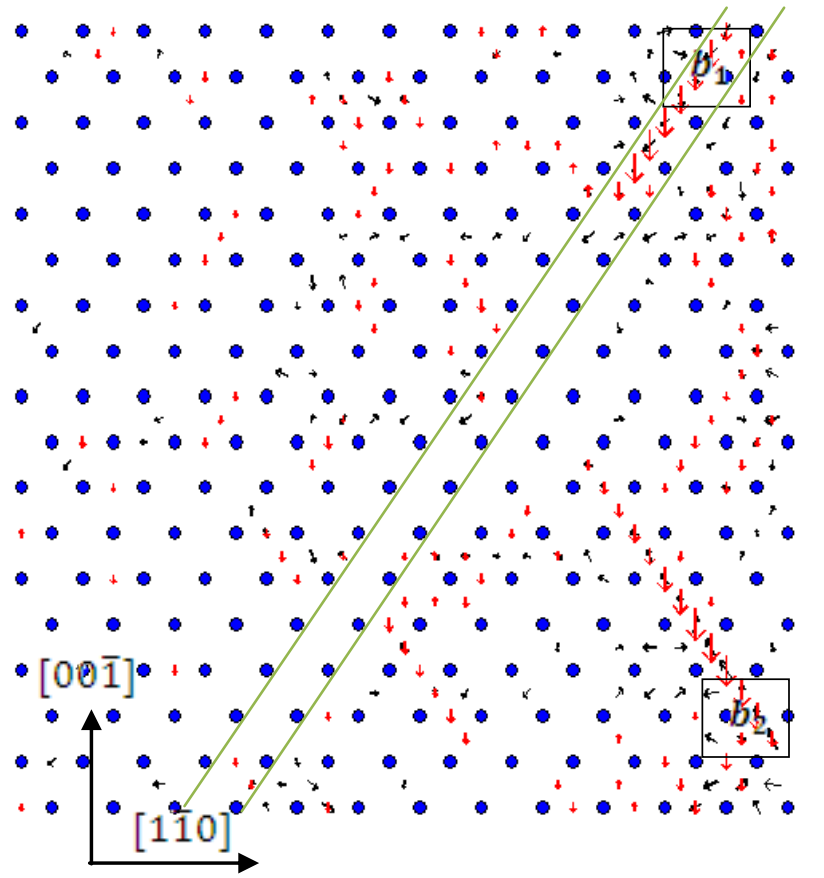

(a) $t=2.7 \mathrm{ps}$

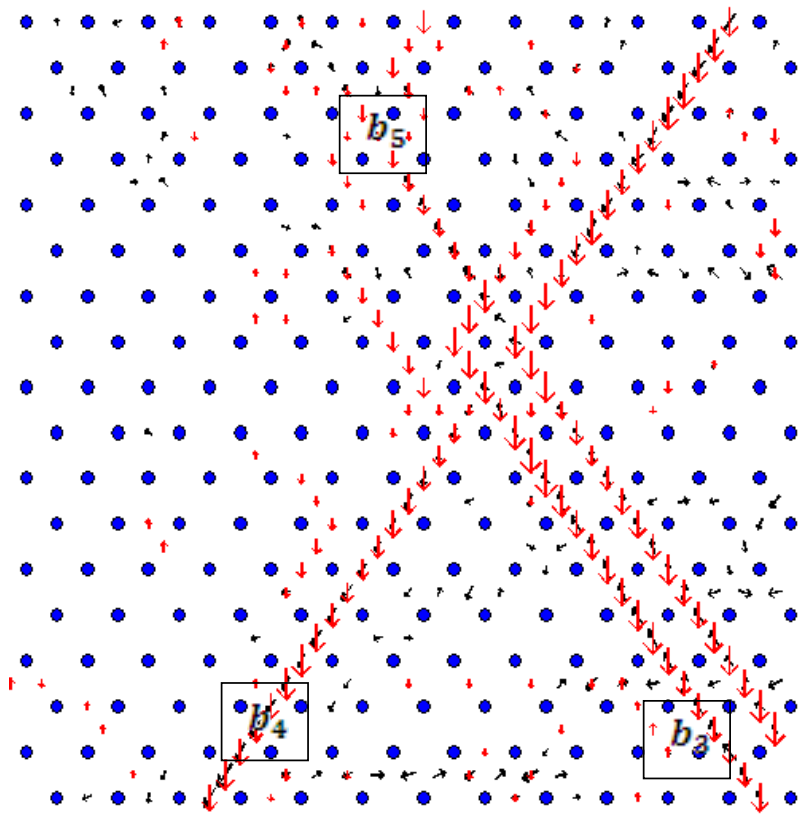

(b) $t=3.6 \mathrm{ps}$

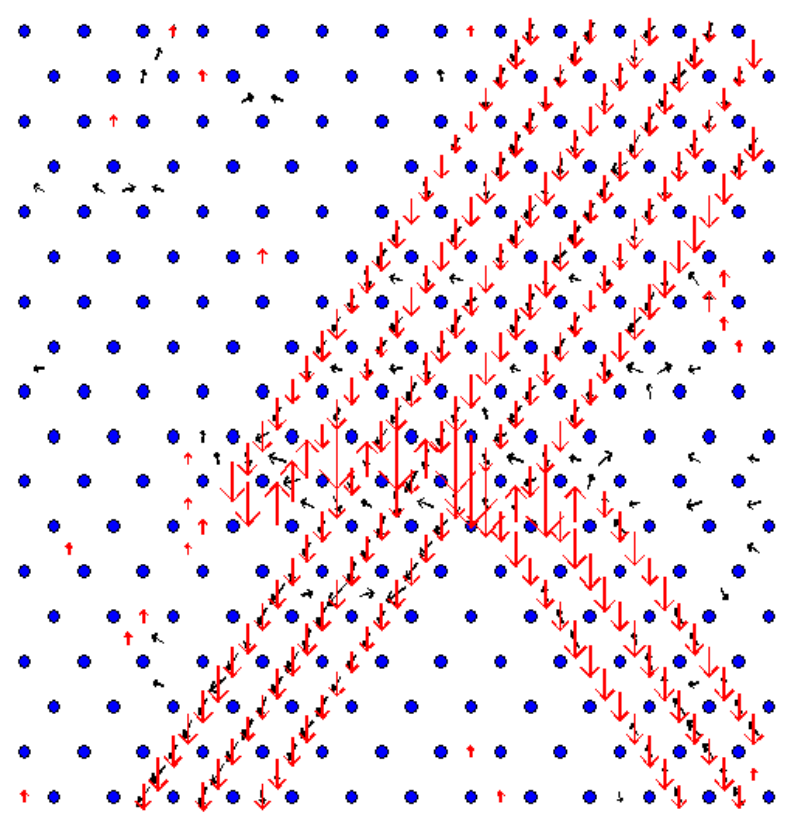

(c) $t=5.4 \mathrm{ps}$

(d) $t=30.0 \mathrm{ps}$ 


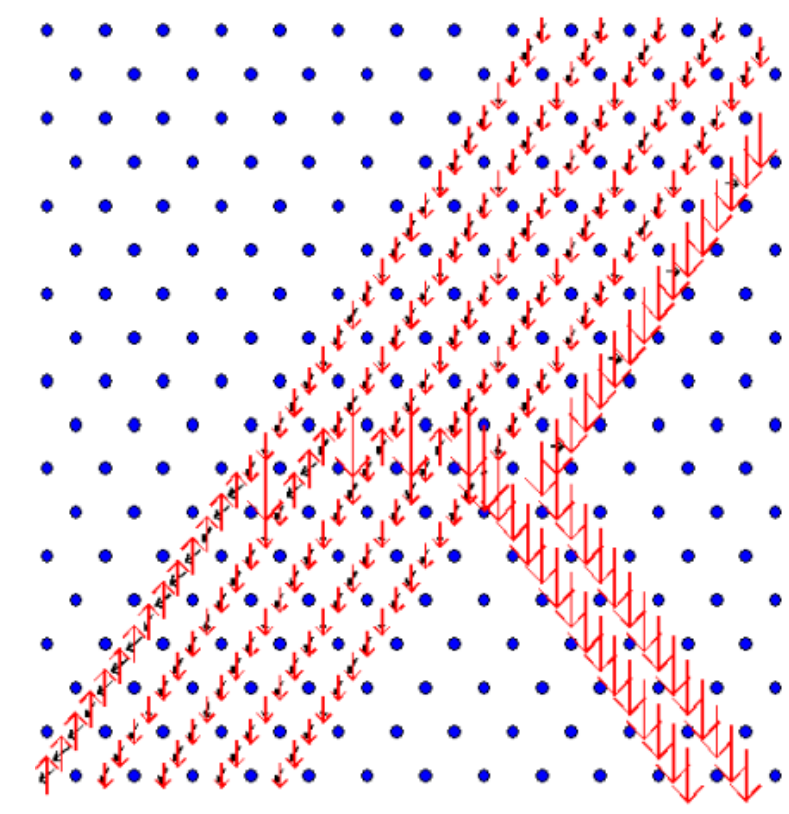

(e) $t=1200.0 \mathrm{ps}$

Fig. 5 Relative displacement map of two (110) planes for the sintering of two aligned cubic initial particles each with 2916 atoms. Time is indicated below each snapshot. The left part of the crystal, in which little deformation occurs, is not drawn to save space. 


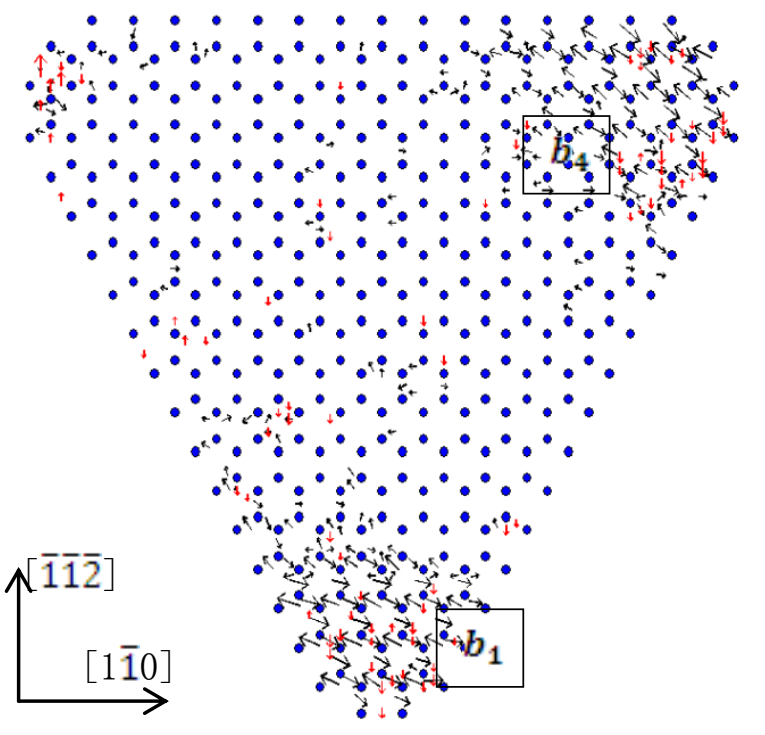

(a) $t=2.7 \mathrm{ps}$

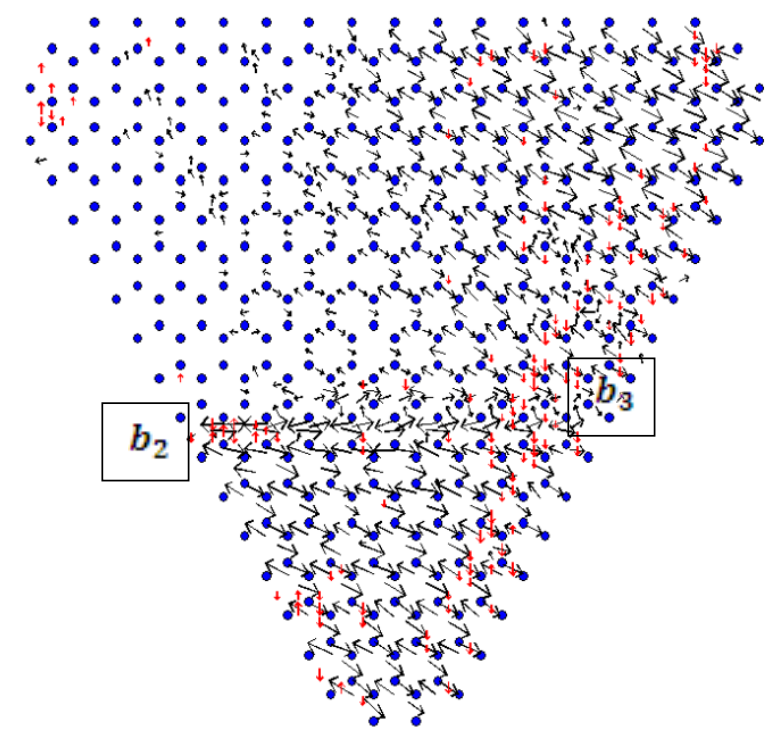

(c) $t=3.3 \mathrm{ps}$

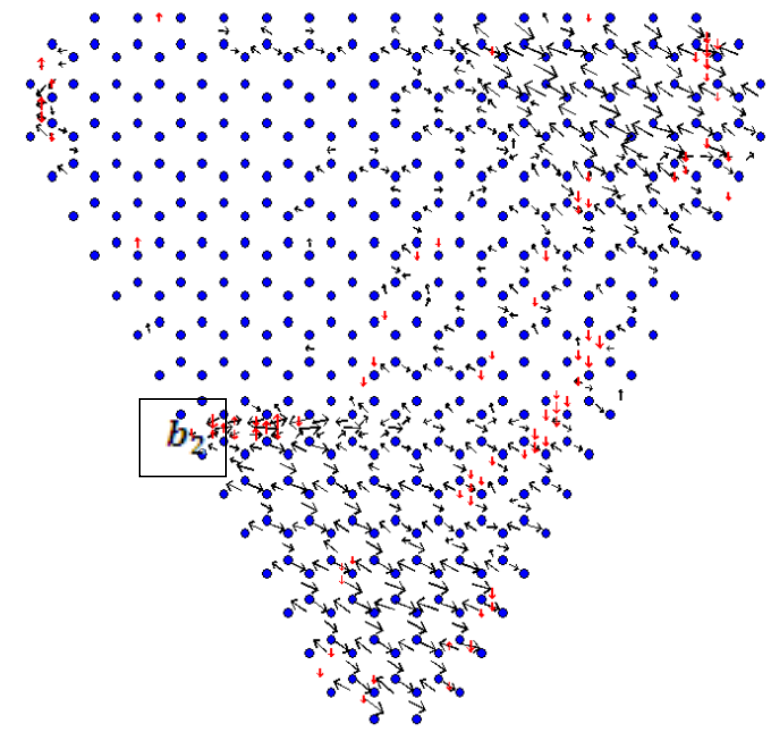

(b) $t=3.0 \mathrm{ps}$

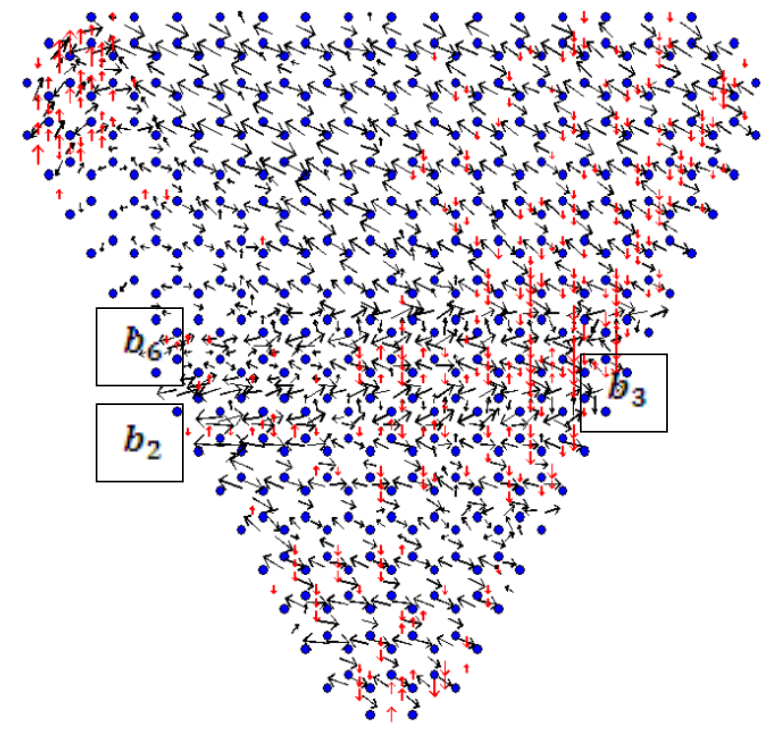

(d) $t=5.1 \mathrm{ps}$

Fig. 6 Relative displacement map of two (111) planes for the case shown in Fig. 5. Time is indicated below each snapshot. 


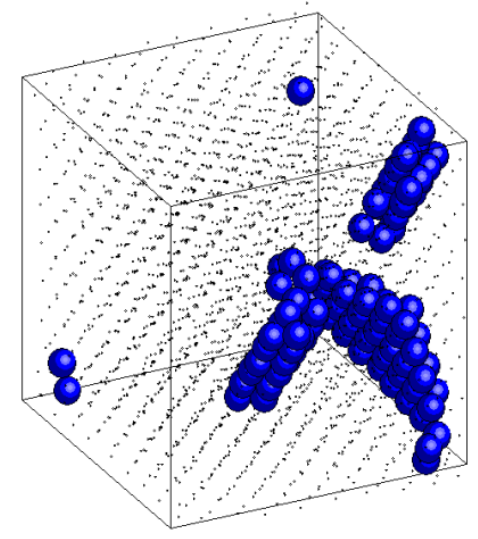

(a) $t=3.0 \mathrm{ps}$

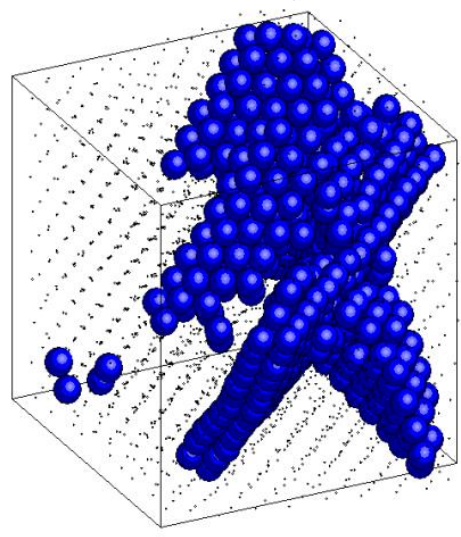

(b) $t=3.9 \mathrm{ps}$

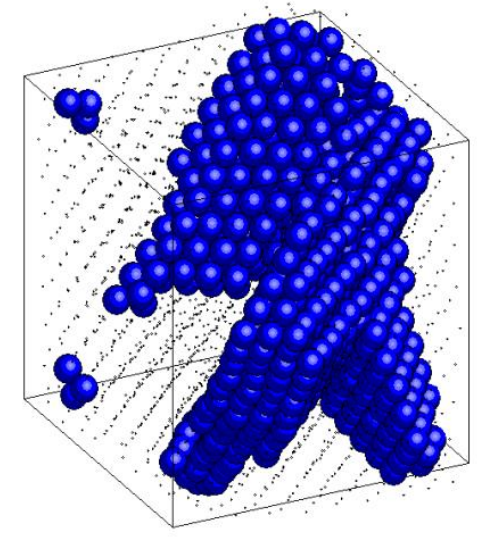

(c) $t=5.1 \mathrm{ps}$

Fig. 7 Crystal structure change during the formation of single twin in the case shown in Figs. 5 and 6. Atoms with large maximum relative displacements are marked by large blue circles. Other atoms are indicated by small black dots. Only atoms belonging to the initial crystal on the negative $\mathrm{x}$ axis are drawn. The box frame indicates the approximate shape of the particle. Time is indicated below each snapshot. 


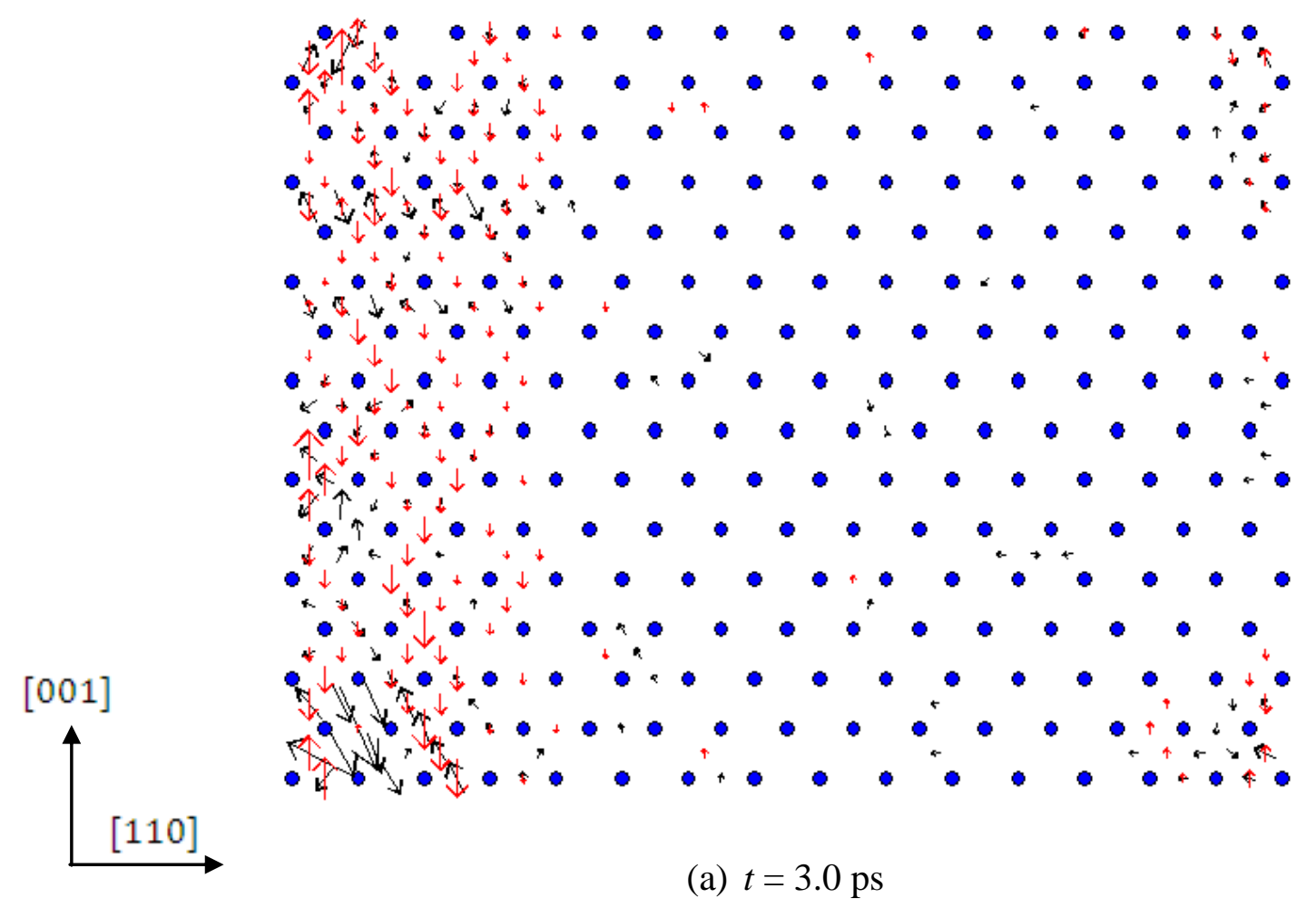

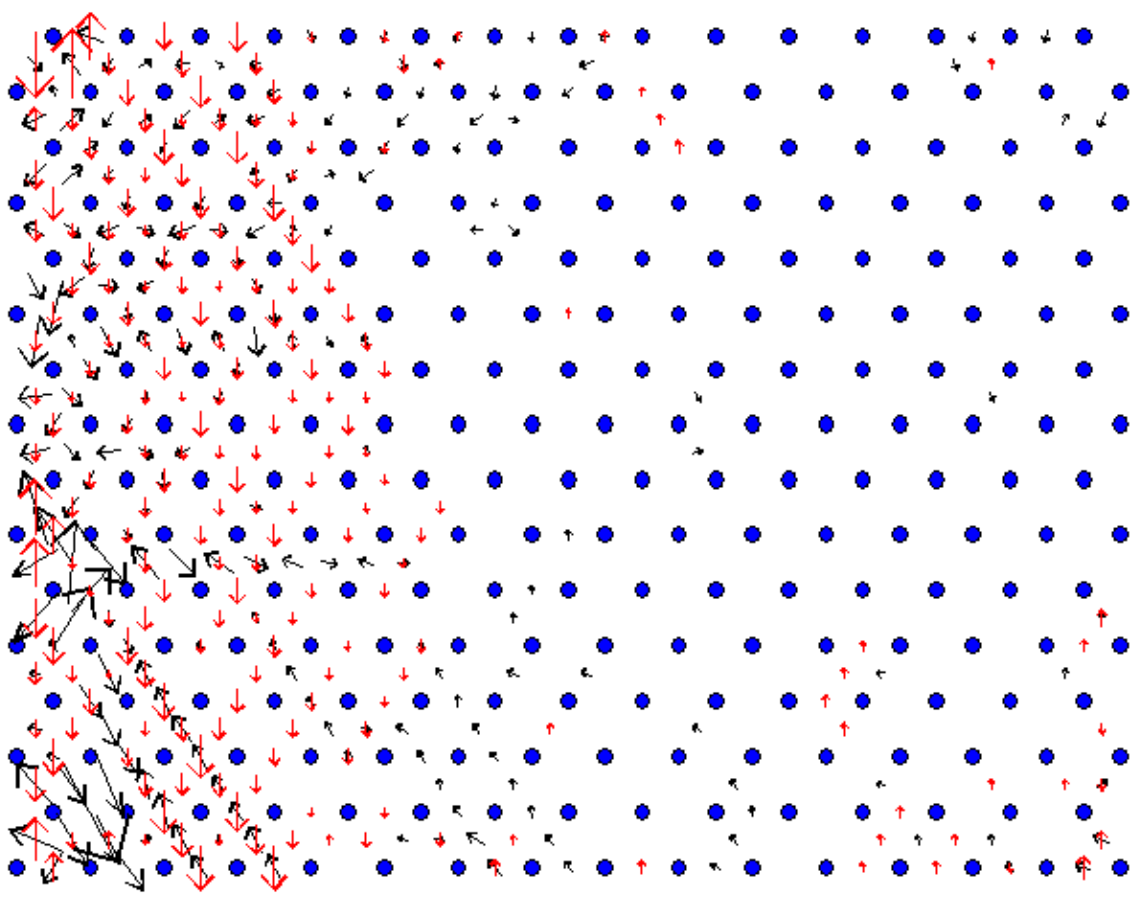

(b) $t=3.6 \mathrm{ps}$ 


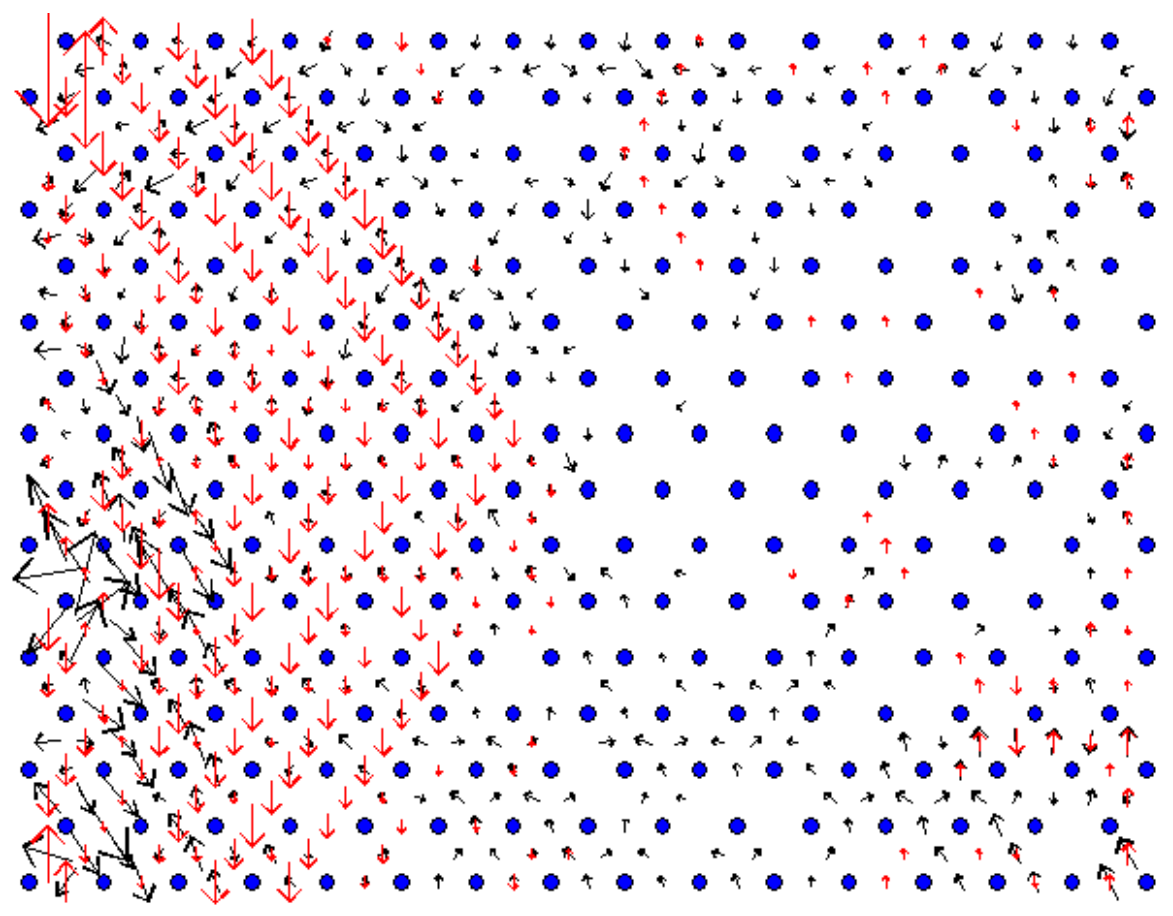

(c) $t=4.5 \mathrm{ps}$

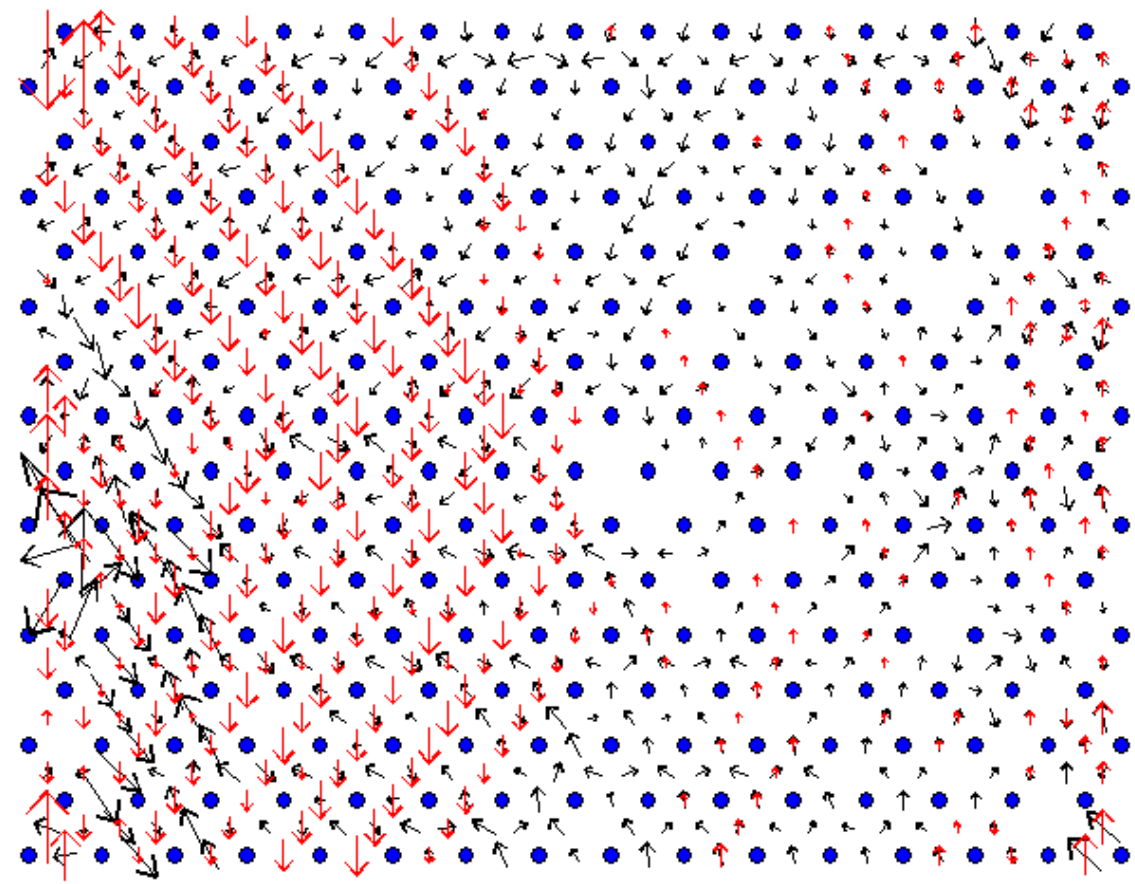

(d) $t=5.1 \mathrm{ps}$ 


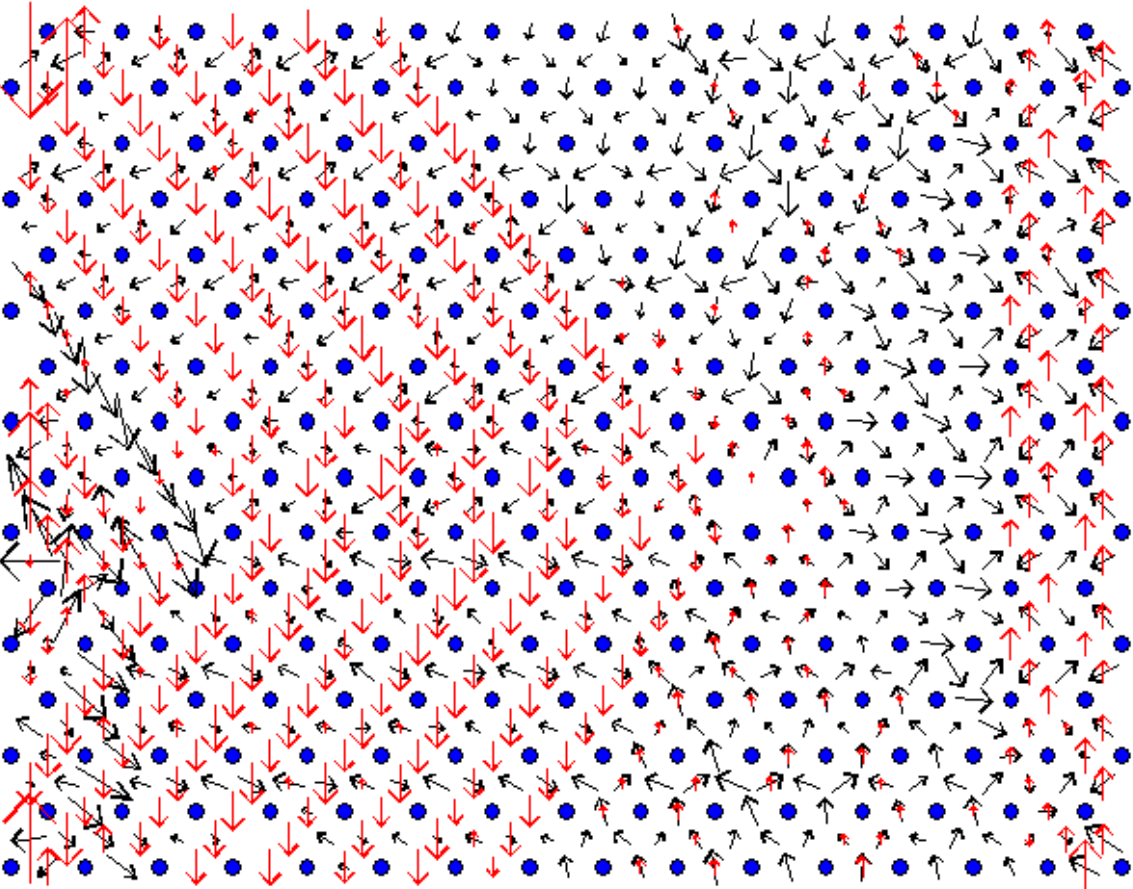

(e) $t=6.0 \mathrm{ps}$

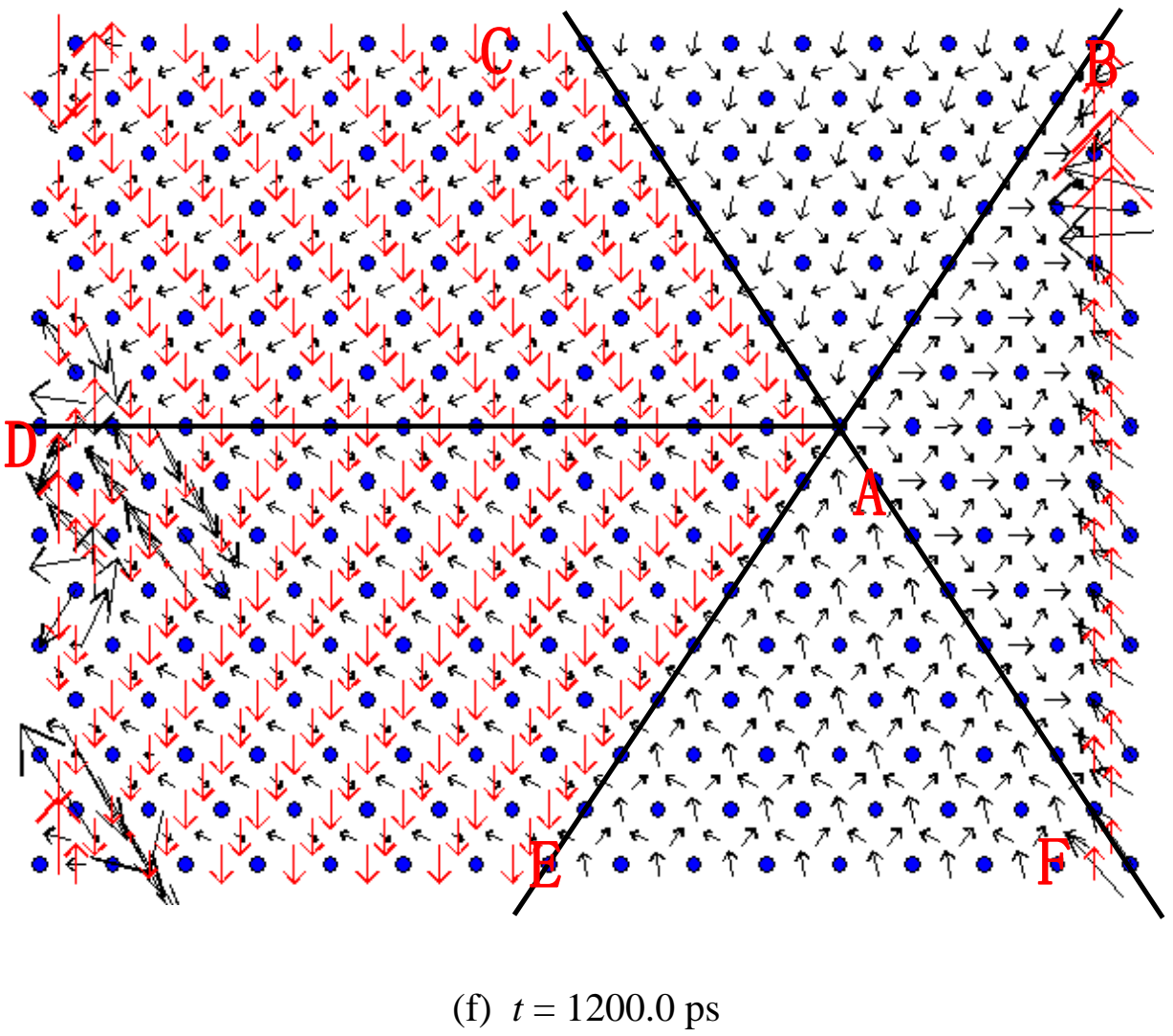


Fig. 8 Relative displacement maps for sintering of two cubic initial particles each with 2048 atoms misoriented by $15^{\circ}$, to form a fivefold twinned structure as in case (f). Time is indicated below each snapshot.

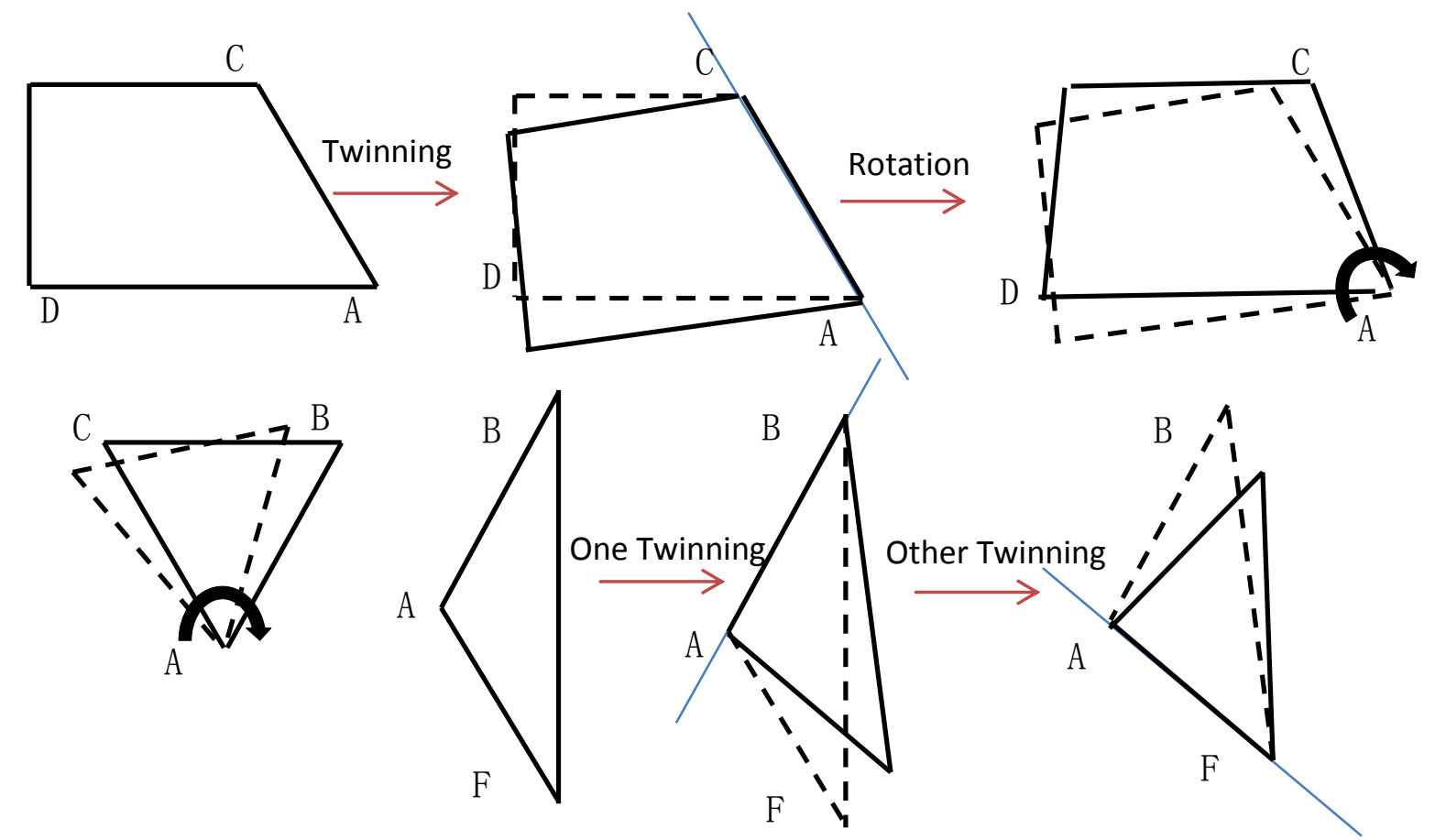

Fig. 9 Schematic illustration for the formation of fivefold twin. Solid frames and dashed frames represent the shape of each part after and before each operation. Blue lines indicate twin boundaries. 


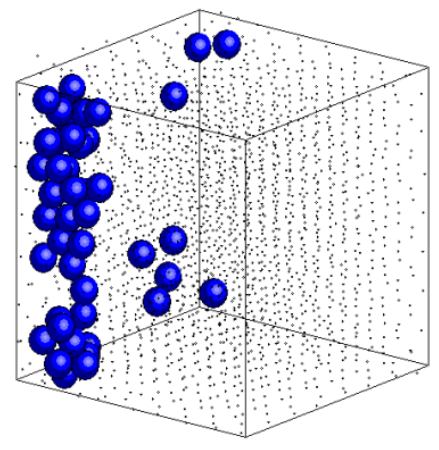

(a) $t=3.0 \mathrm{ps}$

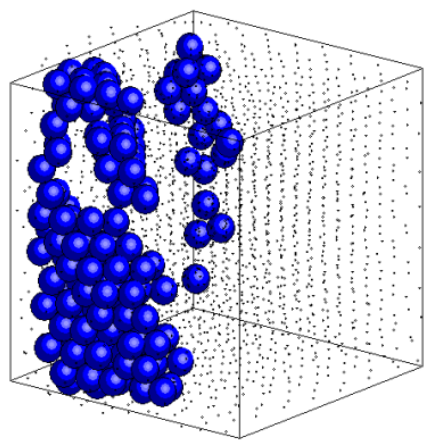

(b) $t=3.9 \mathrm{ps}$

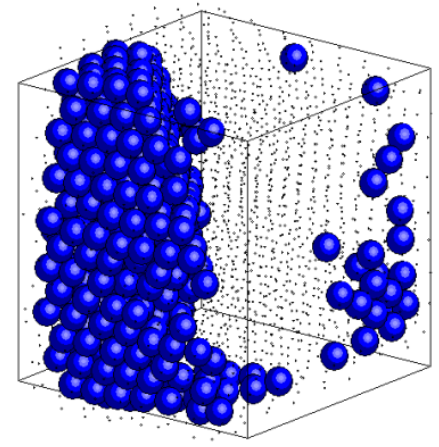

(c) $t=4.8 \mathrm{ps}$

Fig. 10 Crystal structure change during the formation of fivefold twin. Atoms with large maximum relative displacement are marked by large blue circle. Other atoms are indicated by small black dots. Only atoms belonging to the initial crystal on the positive $\mathrm{x}$ axis are drawn. The box frame indicates the approximate shape of the particle. Time is indicated below each snapshot. 